TRANSACTIONS OF THE

AMERICAN MATHEMATICAL SOCIETY

Volume 352, Number 7 , Pages 3065-3101

S 0002-9947(99)02339-9

Article electronically published on October 5, 1999

\title{
A FINITELY AXIOMATIZABLE UNDECIDABLE EQUATIONAL THEORY WITH RECURSIVELY SOLVABLE WORD PROBLEMS
}

\author{
DEJAN DELIĆ
}

\begin{abstract}
In this paper we construct a finitely based variety, whose equational theory is undecidable, yet whose word problems are recursively solvable, which solves a problem stated by G. McNulty (1992). The construction produces a discriminator variety with the aforementioned properties starting from a class of structures in some multisorted language (which may include relations), axiomatized by a finite set of universal sentences in the given multisorted signature.

This result also presents a common generalization of the earlier results obtained by B. Wells (1982) and A. Mekler, E. Nelson, and S. Shelah (1993).
\end{abstract}

\section{InTRODUCTION}

This paper grew out of an attempt to address the following problem, stated in 13]:

Problem 1.1. Is there a finitely based undecidable equational theory with recursively solvable word problems?

We say that a variety $V$ axiomatized by a set $\Sigma$ of identities has recursively solvable word problems (or, simply, that $V$ has solvable word problems), if every finitely presented algebra $\mathbf{A}$ in $V$ has a solvable word problem.

In [14, the authors give an example of a recursively based variety of an infinite signature with the aforementioned properties. Similar examples of a recursively based variety in a finite language were given in [4] and [17.

By strengthening the concept of the recursive solvability of the word problem for a given variety $V$, we arrive at the notion of the uniform solvability of the word problem for $V$. Namely, if we require that there exists a uniform algorithm, which solves the word problem for every finite presentation, relative to $V$, we say that the word problem for $V$ is uniformly solvable.

Trivially, the uniform decidability of the word problem for $V$ implies the recursive solvability of all word problems in $V$; but is the converse true, as well?

Namely, every familiar example of a variety with solvable word problems appeared to be such due to the existence of a uniform algorithm. The classical examples involve abelian groups, abelian semigroups, lattices etc. A comprehensive survey of the known results is given in [13.

Received by the editors June 21, 1996 and, in revised form, January 8, 1998.

1991 Mathematics Subject Classification. Primary 03B25; Secondary 08A50, 08B05.

Key words and phrases. Multisorted logic, universal theory, variety, equational theory, word problem. 
This question was finally answered in a negative way in a paper by A. Mekler, E. Nelson and S. Shelah ([14]), where an intricate way of interpreting a Turing machine with the undecidable halting problem produced a finitely based variety which separates the two levels of solvability of word problem.

It might also be interesting to point out that most of the varieties which are known to have uniformly solvable word problem are such that their uniform word problem is co-NP-complete. These include e.g., distributive lattices, Boolean algebras, varieties generated by a finite nonsolvable group, any finitely generated variety of lattices, etc.

On the other hand, Kozen ([6]) proved that the variety of all algebras in a language containing no relational symbols has uniformly solvable word problem in polynomial time.

A particularly remarkable result from the point of view of computational complexity concerns the variety of commutative semigroups. Namely, Mayr and Meyer (9]) proved that word problems for this variety are polynomial time solvable, yet its uniform word problem is exponential space complete. It is also interesting to point out that the time complexity of the latter problem is not known; it cannot be polynomial, while it can be shown that it is at most double exponential.

A good reference for the computational complexity of the uniform word problem is 2 .

Another aspect of different levels of "recursiveness" of algorithmic problems in varieties of algebras is reflected in the existence of pseudorecursive varieties.

We say that a variety $V$ is pseudorecursive, if its equational theory is undecidable, yet the theory consisting of the equations involving at most $n$ variables, true in $V$, is decidable, for every $n<\omega$.

The existence of finitely based pseudorecursive varieties was first established in [18], again, using a suitable encoding of computations of a Turing machine with the undecidable Halting problem in the equational theory of a variety.

In this paper we provide a positive answer to the problem stated at the very beginning of the paper, i.e., we construct a finitely based variety $V$ whose equational theory is undecidable, yet whose word problem is solvable. This will subsume the main results of [14] and [18, since:

$1^{\circ}$ The theory of quasi-identities of $V$ will be undecidable, which is, according to a result of Mal'cev ([8]), equivalent to the non-existence of a uniform algorithm, which would solve the word problem for any finite presentation relative to $V$. Hence, the uniform word problem for $V$ will be unsolvable, and the main result of [14] follows.

$2^{\circ}$ On the other hand, since in $V$, for every $n<\omega$, the $n$-generated free algebra in $V$ is finitely presented and the solvability of the word problem for this $n$-generated free algebra in $V$ is equivalent to the decidability of the $n$-variable equational theory of $V ; V$ will be pseudorecursive.

Our approach, instead of trying to encode the undecidability of the Halting problem directly into the equations defining $V$, will be to use a class of structures, defined in some multisorted signature (including relations), and then, to translate different (un)decidability properties of that class into the corresponding properties of a variety in a 1-sorted language without relation symbols.

This construction will be driven by the following version of the Halting problem: there is a Turing machine $T$ such that there is no uniform algorithm to decide for which initial configurations $T$ eventually halts; on the other hand, for each $n$, the 
set of initial configurations of length at most $n$ from which $T$ halts is decidable. This nonuniformity for the Halting problem is essentially what makes things work out nicely. For more on the theory of computability and recursive functions, see [1].

One of the main tools throughout this paper will be multisorted logic (with the identity) and we shall freely make use of standard notions and results of it. An introduction to the basic model theory of multisorted structures is given, e.g. in Chapter 5 in [7], and the reader is invited to consult it for the sake of a further reference.

In order to make the exposition more readable, we have decided to deviate from the standard practice of designating different sets of letters for the variables of different sorts. Instead, we use the same standard, letters for variables $(x, y, z, \ldots)$ of all sorts, yet, being almost exclusively interested in sentences, we indicate the sort of a variable by restricting the range of the quantifier in question. For example,

$$
\left(\forall x \in S_{i}\right) \phi(x)
$$

means: "for every $x$ of sort $S_{i}, \phi(x)$ ", while

$$
\left(\exists x \in S_{i}\right) \phi(x)
$$

should be interpreted as: "there exists $x$ of sort $S_{i}$, such that $\phi(x)$ ".

Where the multisorted formula is not a sentence, we indicate explicitly the sort of a variable, as necessary.

A brief discussion on the theory of multisorted varieties can be found in either [16] or [12]. Actually, the techniques developed in section [2] of this paper were inspired by the first part of Chapter 11 in 12, where the authors describe the categorical equivalence between the varieties of multisorted algebras and the varieties in 1sorted algebraic signatures.

The most important tool in our proof is McKenzie's reduction of first order logic to equational logic using discriminator varieties, which is described in [10].

For basic facts from universal algebra and theory of discriminator varieties the reader is referred to [3] and [1].

The most exhaustive reference on algorithmic problems for varieties of algebras is [5], where further information can be found.

Next, we adopt several notational conventions and give some definitions that will be used throughout the paper.

If $a$ is an $n$-tuple from, say, $A_{1} \times A_{2} \times \cdots \times A_{n}$, where $A_{1}, \ldots, A_{n}$ are any sets, the $i$-th component of $a$ will sometimes be referred to as

$$
a^{i} \in A_{i},
$$

or as

$$
a[i] \in A_{i},
$$

depending on which form will be more convenient in a given context.

By a universal theory of a class $\mathcal{K}$ in a (multisorted) signature $\mathcal{L}$, we mean the theory of all universal sentences, in the prenex normal form, true in every structure in $\mathcal{K}$, and it will be denoted by

$$
T h_{\forall}(\mathcal{K}) .
$$

If $\mathcal{K}$ is a (multisorted) class of structures,

$$
T h_{\forall, n}(\mathcal{K})
$$


will denote the fragment of the universal theory of $\mathcal{K}$, consisting of the sentences from $T h_{\forall}(\mathcal{K})$ containing at most $n$ variables of each sort.

Given a (multisorted) class $\mathcal{K}$ of algebras,

$$
T h_{E q, n}(\mathcal{K})
$$

will stand for the set of equations in the signature of $\mathcal{K}$, having at most $n$ variables of each sort.

We say that the (multisorted) class $\mathcal{K}$ is universally pseudorecursive, if $T h_{\forall}(\mathcal{K})$ is undecidable, while $T h_{\forall, n}(\mathcal{K})$ is decidable, for every $n<\omega$.

Similarly, the class $\mathcal{K}$ is said to be pseudorecursive, if $T h_{E q}(\mathcal{K})$, the equational theory of $\mathcal{K}$ is undecidable, while all $T h_{E q, n}(\mathcal{K}), n$-variable fragments of the equational theory, are decidable.

Section 2 constitutes the proof of the following theorem.

Theorem 1.2. Given a universally pseudorecursive class $\mathcal{K}$ of multisorted structures axiomatized by a finite set $\Phi$ of universal sentences in a multisorted language $\mathcal{L}$, one can construct a finitely based pseudorecursive discriminator variety $\mathcal{V}$ whose word problems are solvable.

\section{From a multisorted universal Class to A PSEUdorecursive VARIETy}

The idea of the proof of Theorem 1.2 is the following:

(1) Eliminate the relation symbols. Given a universally pseudorecursive class $\mathcal{K}$ of multisorted structures in a language $\mathcal{L}$, axiomatized by a finite set $\Phi$ of universal sentences, construct a universally pseudorecursive multisorted class $\mathcal{K}^{*}$, in a language $\mathcal{L}^{*}$, which does not contain relation symbols, and which is axiomatized by a finite set of universal sentences $\Psi$ in $\mathcal{L}^{*}$.

(2) Eliminate the constant symbols. Given a universally pseudorecursive class $\mathcal{K}$ of multisorted structures in a language $\mathcal{L}$, which does not contain relation symbols, and which is axiomatized by a finite set $\Phi$ of universal sentences in $\mathcal{L}$, construct a universally pseudorecursive multisorted class $\mathcal{K}^{*}$ in a language $\mathcal{L}^{*}$, which does not contain either relation or constant symbols, and which is axiomatized by a finite set $\Psi$ of universal sentences in $\mathcal{L}^{*}$.

(3) Reduce to a single sort. Given a universally pseudorecursive class $\mathcal{K}$ of multisorted structures in a language $\mathcal{L}$, not containing any relation or constant symbols, and axiomatized by a finite set $\Psi$ of universal sentences in $\mathcal{L}$, construct a universally pseudorecursive class of structures $\mathcal{K}^{*}$ in an ordinary 1 -sorted algebraic language $\mathcal{L}^{*}$, such that $\mathcal{K}^{*}$ is axiomatized by a finite set of universal sentences.

(4) Reduce to an equational class. Given a universally pseudorecursive class of algebras $\mathcal{K}$, axiomatized by a finite set of universal sentences in $\mathcal{L}$, the associated discriminator variety denoted $\mathbf{V}\left(\mathcal{K}^{t}\right)$ is a finitely based pseudorecursive variety in the language $\mathcal{L}^{t}$, whose word problems are solvable.

The general method that will be used in steps (1)-(3) will depend on the following definition:

Definition 2.1. Let $\mathcal{L}$ and $\mathcal{L}^{*}$ be two multisorted languages, and $\Sigma$ a finite set of universal sentences in $\mathcal{L}^{*}$. The system of transformations $\langle *, \Sigma\rangle$ is called a $\Sigma$ equivalence system if it satisfies the following conditions: 
1. For any structure $\mathbf{A}$ of signature $\mathcal{L}, \mathbf{A}^{*}$ is a structure of signature $\mathcal{L}^{*}$, which satisfies $\Sigma$. Conversely, for any structure $\mathbf{B}$ of signature $\mathcal{L}^{*}$, satisfying $\Sigma, \mathbf{B}_{*}$ is a structure of signature $\mathcal{L}$, so that

$$
\left(\mathbf{A}^{*}\right)_{*} \cong \mathbf{A},
$$

and

$$
\left(\mathbf{B}_{*}\right)^{*} \cong \mathbf{B} .
$$

2. For any sentence $\phi$ in $\mathcal{L}$, sentence $\phi^{*}$ is a sentence in $\mathcal{L}^{*}$ constructed effectively from $\phi$, and, conversely, for any sentence $\psi$ in $\mathcal{L}^{*}, \psi_{*}$ is a sentence constructed effectively from $\psi$ in $\mathcal{L}$, so that, for any structure $\mathbf{A}$ of signature $\mathcal{L}$, any structure $\mathbf{B}$ of signature $\mathcal{L}^{*}$ satisfying $\Sigma$, and $\phi, \psi$ sentences in $\mathcal{L}, \mathcal{L}^{*}$, respectively, we have the following:

$$
\mathbf{A} \models \phi \quad \text { iff } \quad \mathbf{A}^{*} \models \phi^{*},
$$

and

$$
\mathbf{B} \models \psi \quad \text { iff } \quad \mathbf{B}_{*} \models \psi_{*} .
$$

Moreover, these two transformations are such that they carry universal sentences into universal sentences.

Then, $\mathcal{K}^{*}$ is defined to be the class

$$
\mathcal{K}^{*}=\mathbf{I}\left\{\mathbf{A}^{*}: \mathbf{A} \in \mathcal{K}\right\}
$$

where $\mathbf{I}$ denotes the closure of the class under isomorphisms.

Definition 2.2. Suppose that the language $\mathcal{L}$ is $k$-sorted, with the sorts $S_{1}, \ldots, S_{k}$. The transformation $\psi \rightarrow \psi_{*}$, which, given a universal sentence $\psi$ in $\mathcal{L}^{*}$ associates to it a universal sentence $\psi_{*}$ in $\mathcal{L}$, is said to be variable bounded if and only if there are functions $\theta_{1}, \ldots, \theta_{k}$ on the natural numbers such that for every formula $\psi$, if $\psi$ has at most $n$ variables of each sort, then $\psi_{*}$ has at most $\theta_{i}(n)$ variables of sort $S_{i}$, for each $i=1, \ldots, k$.

If a $\Sigma$-equivalence system $\langle *, \Sigma\rangle$ is such that its transformation function on the sentences from $\mathcal{L}$ into $\mathcal{L}^{*}$ is variable-bounded, then $\langle *, \Sigma\rangle$ will be said to be variablebounded, as well.

In that case, the following theorem will be true:

Theorem 2.3. If $\mathcal{K}$ is a universally pseudorecursive class axiomatized by a set $\Phi$ of universal sentences, and $\langle *, \Sigma\rangle$ is a variable-bounded $\Sigma$-equivalence system, then $\mathcal{K}^{*}$ is a universally pseudorecursive class axiomatized by $\Phi^{*} \cup \Sigma$, where

$$
\Phi^{*}=\left\{\phi^{*}: \phi \in \Phi\right\} .
$$

Proof. It is quite a straightforward exercise to show that $\mathcal{K}^{*}$ is axiomatized by $\Phi^{*} \cup \Sigma$, and that $T h_{\forall}\left(\mathcal{K}^{*}\right)$ is undecidable.

Let $\psi$ be a universal sentence in $\mathcal{L}^{*}$, which contains at most $n$ variables of each sort, and let $\theta_{1}, \ldots, \theta_{k}$ be the functions which witness variable-boundedness. Consider

$$
m=\max \left\{\theta_{1}(n), \theta_{2}(n), \ldots, \theta_{k}(n)\right\} .
$$

Then,

$$
\psi \in T h_{\forall, n}\left(\mathcal{K}^{*}\right) \quad \text { iff } \quad \psi_{*} \in T h_{\forall, m}(\mathcal{K}) .
$$

Since $T h_{\forall, m}(\mathcal{K})$ is decidable, so is $T h_{\forall, n}\left(\mathcal{K}^{*}\right)$. 
This concludes our preliminary discussion on how steps (1)-(3) will be carried out. In the sequel, we shall refer to these results and confine ourselves to defining $\mathcal{L}^{*}, \Sigma$ and the corresponding transformations for each particular case.

2.1. Getting rid of relations. Suppose $\mathcal{L}$ is a $k$-sorted language with sorts $S_{1}, \ldots, S_{k}$. We define $\mathcal{L}^{*}$ to be a $(k+1)$-sorted language whose sorts are those of $\mathcal{L}$, plus a new sort $S_{k+1}$, which is the sort of two new constant symbols 0 and 1 .

Also, $\mathcal{L}^{*}$ retains all function and constant symbols from $\mathcal{L}$, but the relation symbols of $\mathcal{L}$ are replaced by function symbols intended to denote their characteristic functions in the following way: if $\rho$ is an $m$-ary relation symbol of $\mathcal{L}$,

$$
\rho \subseteq S_{i_{1}} \times \cdots \times S_{i_{m}},
$$

then $\rho$ is replaced by an $m$-ary function symbol $R$ in $\mathcal{L}^{*}$, so that

$$
R: S_{i_{1}} \times \cdots \times S_{i_{m}} \mapsto S_{k+1} .
$$

Let $\Sigma$ consist of the following universal sentences:

$$
\begin{gathered}
\left(\forall x \in S_{k+1}\right)(x=0 \vee x=1), \\
0 \neq 1 .
\end{gathered}
$$

The transformation on the level of structures is now obvious and therefore will be omitted.

Next, we define the corresponding transformations on the sentences in $\mathcal{L}$ and $\mathcal{L}^{*}$.

Let $\phi$ be a sentence in $\mathcal{L}$. Replace every atomic subformula of $\phi$ of the form

$$
\rho\left(t_{1}(\bar{x}), \ldots, t_{m}(\bar{x})\right),
$$

where

$$
\rho \subseteq S_{i_{1}} \times \cdots \times S_{i_{m}},
$$

is a relation and $t_{j}(\bar{x})$ is a term of the sort $S_{i_{j}}$, for $1 \leqslant j \leqslant m$ by

$$
R\left(t_{1}(\bar{x}), \ldots, t_{m}(\bar{x})\right)=1,
$$

where $R$ is the corresponding function symbol in $\mathcal{L}^{*}$. Denote the formula in $\mathcal{L}^{*}$, obtained from $\phi$ in this way by $\phi^{*}$.

Now, suppose $\psi$ is a sentence in $\mathcal{L}^{*}$, which can be assumed to be in prenex normal form. If $\psi$ is of the form

$$
Q \bar{x}\left(\forall z \in S_{k+1}\right) \vartheta(\bar{x}, z),
$$

where $Q \bar{x}$ is a quantifier prefix in $\psi$, this formula will be equivalent, modulo $\Sigma$, to

$$
Q \bar{x}(\vartheta(\bar{x}, 0) \wedge \vartheta(\bar{x}, 1)) ;
$$

while, if $\psi$ is of the form

$$
Q \bar{x}\left(\exists z \in S_{k+1}\right) \vartheta(\bar{x}, z),
$$

it will be equivalent to

$$
Q \bar{x}(\vartheta(\bar{x}, 0) \vee \vartheta(\bar{x}, 1)) .
$$

Hence, after putting each of (2.1), (2.2) into prenex normal form, we may assume that $\psi$ does not contain variables of sort $S_{k+1}$.

Thus, the atomic subformulas of $\psi$, which involve terms of the sort $S_{k+1}$, are of one of the following forms. 
(1) $R\left(t_{1}(\bar{x}), \ldots, t_{m}(\bar{x})\right)=1$

where

$$
R: S_{i_{1}} \times \cdots \times S_{i_{m}} \rightarrow S_{k+1}
$$

and every $t_{j}(\bar{x})$ is a term of the sort $S_{i_{j}}$.

(2) $R\left(t_{1}(\bar{x}), \ldots, t_{m}(\bar{x})\right)=0$

where

$$
R: S_{i_{1}} \times \cdots \times S_{i_{m}} \rightarrow S_{k+1}
$$

and every $t_{j}(\bar{x})$ is a term of the sort $S_{i_{j}}$.

(3) $R_{1}\left(t_{1}(\bar{x}), \ldots, t_{m}(\bar{x})\right)=R_{2}\left(u_{1}(\bar{x}), \ldots, u_{n}(\bar{x})\right)$.

(4) $0=0$

(5) $1=1$.

(6) $0=1$.

If an atomic subformula of $\psi$ is of one of the forms (1) or (2), we replace it by

$$
\rho\left(t_{1}(\bar{x}), \ldots, t_{m}(\bar{x})\right)
$$

or

$$
\neg \rho\left(t_{1}(\bar{x}), \ldots, t_{m}(\bar{x})\right),
$$

respectively, where $\rho$ is the relation symbol in $\mathcal{L}$ corresponding to $R$.

When an atomic subformula of $\psi$ is of the form (3), we first replace it by

$$
\begin{aligned}
& \left(R_{1}\left(t_{1}(\bar{x}), \ldots, t_{m}(\bar{x})\right)=0 \wedge R_{2}\left(u_{1}(\bar{x}), \ldots, u_{n}(\bar{x})\right)=0\right) \\
& \vee\left(R_{1}\left(t_{1}(\bar{x}), \ldots, t_{m}(\bar{x})\right)=1 \wedge R_{2}\left(u_{1}(\bar{x}), \ldots, u_{n}(\bar{x})\right)=1\right),
\end{aligned}
$$

and then handle this formula as in the previous case.

The construction of $\psi_{*}$ with the required properties is now easy and is left as an exercise to the reader.

2.2. Elimination of constants. Throughout this part, we assume that the class $\mathcal{K}$ of structures in the language $\mathcal{L}$, which does not include relation symbols, is universally pseudorecursive and axiomatized by a finite set $\Phi$ of universal sentences in $\mathcal{L}$. Let $S_{1}, \ldots, S_{k}$ be the sorts of $\mathcal{L}$.

Let $\mathcal{L}^{*}$ be the language with the same sorts as $\mathcal{L}$, obtained from $\mathcal{L}$ by replacing all the constant symbols $c \in \mathcal{L}$ of sort $S_{i}$ by the corresponding unary function symbols

$$
f_{c}: S_{i} \rightarrow S_{i}
$$

Let $\Sigma$ be the set of the following universal sentences in $\mathcal{L}^{*}$ :

$$
\left(\forall x \in S_{i}\right)\left(\forall y \in S_{i}\right)\left(f_{c}(x)=f_{c}(y)\right), \quad c \in \mathcal{L} \backslash \mathcal{L}^{*} .
$$

If $\mathbf{A}$ is a structure of signature $\mathcal{L}$, let $\mathbf{A}^{*}$ be the structure with the same universe as $\mathbf{A}$, such that, for every function symbol $g \in \mathcal{L} \cap \mathcal{L}^{*}$,

$$
g^{\mathbf{A}}=g^{\mathbf{A}^{*}},
$$

and, for $f_{c} \in \mathcal{L}^{*} \backslash \mathcal{L}$, where $c$ is of the sort $S_{i}$, let

$$
f_{c}: S_{i} \rightarrow S_{i}
$$

be such that $f_{c}^{\mathbf{A}^{*}}$ has the constant value $c^{\mathbf{A}}$. 
On the other hand, if $\mathbf{B}$ is any structure of signature $\mathcal{L}^{*}$, which satisfies $\Sigma$, define $\mathbf{B}_{*}$ to be the structure of signature $\mathcal{L}$, whose universe is that of $\mathbf{B}$, and for $g \in \mathcal{L} \cap \mathcal{L}^{*}$,

$$
g^{\mathbf{B}_{*}}=g^{\mathbf{B}},
$$

while, for $f_{c} \in \mathcal{L}^{*} \backslash \mathcal{L}$, the corresponding $c \in \mathcal{L}$ will be interpreted in $\mathbf{B}_{*}$ as the constant value of $f_{c}^{\mathrm{B}}$.

It is straightforward to check that, for any structure $\mathbf{A}$ of signature $\mathcal{L}$, and any B of signature $\mathcal{L}^{*}$, which satisfies the universal sentences in $\Sigma$,

$$
\left(\mathbf{A}^{*}\right)_{*} \cong \mathbf{A}
$$

and

$$
\left(\mathbf{B}_{*}\right)^{*} \cong \mathbf{B} .
$$

Given a formula $\phi(\bar{x})$ in $\mathcal{L}$, let $\phi^{\prime}(\bar{x}, \bar{u})$ be a formula obtained from $\phi(\bar{x})$ by replacing each occurrence of $c \in \mathcal{L}$ of the sort $S_{i}$ with a variable $u$ of the same sort which does not occur freely in $\phi(\bar{x})$. We assume that a systematic, effective way to choose such variables is set up in advance. Finally, let $\phi^{*}(\bar{x})$ be $\forall \bar{u} \phi^{\prime}(\bar{x}, \bar{u})$.

Proposition 2.4. Let $\mathbf{A}$ be a structure of signature $\mathcal{L}, \phi(\bar{x})$ a formula in $\mathcal{L}$, and $\bar{a}$ a tuple of elements of $A$ of the appropriate sorts. Then, for all $b_{1} \in S_{1}^{\mathbf{A}}, \ldots, b_{k} \in S_{k}^{\mathbf{A}}$,

$$
\mathbf{A} \models \phi(\bar{a}) \quad \text { iff } \quad \mathbf{A}^{*} \models \phi^{\prime}(\bar{a}, \bar{b}) .
$$

Corollary 2.5. If $\mathbf{A}$ is a structure of signature $\mathcal{L}$, and $\phi$ a sentence in $\mathcal{L}$, then

$$
\mathbf{A} \models \phi \quad \text { iff } \quad \mathbf{A}^{*} \models \phi^{*} .
$$

If $t(\bar{x})$ is a term in $\mathcal{L}^{*}$, which contains an occurrence of some $f_{c} \in \mathcal{L}^{*} \backslash \mathcal{L}$, and if $f_{c}(u(\bar{x}))$ is a subterm of $t(\bar{x})$, which is not nested inside any other subterm of $t(\bar{x})$ of the form $f_{d}(v(\bar{x}))$, where $f_{d} \in \mathcal{L}^{*} \backslash \mathcal{L}$, then replace $f_{c}(u(\bar{x}))$ by the corresponding $c \in \mathcal{L} \backslash \mathcal{L}^{*}$.

Now, given a formula $\psi(\bar{x})$ in $\mathcal{L}^{*}$, define $\psi_{*}(\bar{x})$ to be the formula in $\mathcal{L}$, which is obtained from $\psi(\bar{x})$ by transforming each term $t(\bar{x})$ occurring in $\psi(\bar{x})$ in the above described manner, plus deleting all the quantifiers that refer to the variables eliminated in this process.

Hence, we have the following proposition, whose proof is straightforward:

Proposition 2.6. For a structure $\mathbf{B}$ of signature $\mathcal{L}^{*}$, which satisfies the universal sentences in $\Sigma$, a formula $\psi(\bar{x})$ in $\mathcal{L}^{*}$, and a tuple $\bar{b}$ of elements of $\mathbf{B}$ of the appropriate sorts,

$$
\mathbf{B}=\psi(\bar{b}) \quad \text { iff } \quad \mathbf{B}_{*}=\psi_{*}(\bar{b}) .
$$

This transformation does not increase the number of the variables of any sort in $\psi$, so that the class $\mathcal{K}^{*}$ will have all the desired properties.

2.3. From the multisorted to a 1 -sorted class. In this part, we make the transition from multisorted to 1-sorted structures. The standard approach of taking the disjoint union of the sorts (an early reference is [15]) will not be used here; instead we opt for the technique developed in [12] for classes of unary algebras, and put it in a more general setting.

Let $\mathcal{K}$ be a multisorted class of algebras of signature $\mathcal{L}$, axiomatized by a finite set $\Phi$ of universal sentences in $\mathcal{L}$, and which is universally pseudorecursive. $\mathcal{L}$ is 
also assumed not to contain any relation or constant symbols, and its sorts will be denoted by $S_{1}, S_{2}, \ldots, S_{n}$.

The type of a term in $\mathcal{L}$ is defined in a usual way. If the type of the term $t\left(x_{1}, \ldots, x_{k}\right)$ is

$$
\left\langle S_{i_{1}}, \ldots, S_{i_{k}}, S_{j}\right\rangle
$$

$S_{j}$ will be referred to as the sort of $t$.

We define the signature $\mathcal{L}^{*}$ in the following way: If

$$
f: S_{i_{1}} \times \cdots \times S_{i_{k}} \rightarrow S_{j}
$$

is a $k$-ary operation symbol in $\mathcal{L}$, we define $f^{*}$ to be a $k$-ary operation symbol in $\mathcal{L}^{*}$. Besides the operation symbols $f^{*}$ corresponding to $f \in \mathcal{L}$, the language $\mathcal{L}^{*}$ will contain an $n$-ary function symbol $d$.

Let $\Sigma$ consist of the following identities in $\mathcal{L}^{*}$ :

$$
\begin{gathered}
d(x, x, \ldots, x)=x, \\
d\left(d\left(\overline{x_{1}}\right), d\left(\overline{x_{2}}\right), \ldots, d\left(\overline{x_{n}}\right)\right)=d\left(x_{1}^{1}, x_{2}^{2}, \ldots, x_{n}^{n}\right),
\end{gathered}
$$

where $\bar{x}_{i}=\left\langle x_{1}^{i}, \ldots, x_{n}^{i}\right\rangle$ is an $n$-tuple of variables in $\mathcal{L}^{*}$, and

$$
f^{*}\left(d\left(\overline{x_{1}}\right), \ldots, d\left(\overline{x_{k}}\right)\right)=d\left(x_{1}^{1}, \ldots, x_{j-1}^{1}, f^{*}\left(x_{i_{1}}^{1}, \ldots, x_{i_{k}}^{k}\right), x_{j+1}^{1}, \ldots, x_{n}^{1}\right)
$$

for $f \in \mathcal{L} k$-ary of type $\left\langle S_{i_{1}}, \ldots, S_{i_{k}}, S_{j}\right\rangle$.

Given a multisorted algebra

$$
\mathbf{A}=\left(S_{1}^{\mathbf{A}}, \ldots, S_{n}^{\mathbf{A}}\right)
$$

of signature $\mathcal{L}$, we define an algebra $\mathbf{A}^{*}$ of signature $\mathcal{L}^{*}$ in the following way:

- the universe of $\mathbf{A}^{*}$ is

$$
S_{1}^{\mathbf{A}} \times \cdots \times S_{n}^{\mathbf{A}}
$$

- if $f \in \mathcal{L}$ is $k$-ary of the type $\left\langle S_{i_{1}}, \ldots, S_{i_{k}}, S_{j}\right\rangle$, and $a_{1}, \ldots, a_{k} \in A^{*}$ such that

$$
a_{i}=\left\langle a_{1}^{i}, \ldots, a_{n}^{i}\right\rangle, \quad a_{r}^{i} \in S_{r}^{i}(1 \leqslant r \leqslant n),
$$

then

$$
\left(f^{*}\right)^{\mathbf{A}^{*}}:\left(A^{*}\right)^{n} \rightarrow A^{*}
$$

is defined by

$$
\left(f^{*}\right)^{\mathbf{A}^{*}}\left(a_{1}, \ldots, a_{k}\right)=\left\langle a_{1}^{1}, a_{2}^{1}, \ldots, a_{j-1}^{1}, f^{\mathbf{A}}\left(a_{i_{1}}^{1}, \ldots, a_{i_{k}}^{k}\right), a_{j+1}^{1}, \ldots, a_{n}^{1}\right\rangle ;
$$

- $d^{\mathbf{A}^{*}}$ is the natural $n$-ary decomposition operation on $\mathbf{A}^{*}$, given by

$$
d^{\mathbf{A}^{*}}\left(a_{1}, \ldots, a_{n}\right)=\left\langle a_{1}^{1}, a_{2}^{2}, \ldots, a_{n}^{n}\right\rangle .
$$

It is a rather straightforward exercise to verify that, in any $\mathbf{A}^{*}$ defined in this way, all the identities in $\Sigma$ are satisfied.

Now, given any algebra $\mathbf{B}$ of signature $\mathcal{L}^{*}$, which satisfies $\Sigma$, we show how to construct the corresponding $\mathbf{B}_{*}$.

Since $\mathbf{B}$ satisfies (2.3), (2.4),$d^{\mathbf{B}}$ will be the natural $n$-ary decomposition operation on $B$ (see [12]). In other words, there exist $B_{1}, \ldots, B_{n}$ such that $B$ is in a bijective correspondence with $B_{1} \times \cdots \times B_{n}$ via the bijection

$$
\iota: B \rightarrow B_{1} \times \cdots \times B_{n} \text {; }
$$


then,

$$
d^{\mathbf{B}}\left(b_{1}, \ldots, b_{n}\right)=\iota^{-1}\left(\iota\left(b_{1}\right)[1], \ldots, \iota\left(b_{n}\right)[n]\right) .
$$

For the sake of simplicity, we shall identify $B$ with $B_{1} \times \cdots \times B_{n}$, i.e., we assume that

$$
B=B_{1} \times \cdots \times B_{n}
$$

and

$$
d^{\mathbf{B}}\left(b_{1}, \ldots, b_{n}\right)=\left(b_{1}[1], \ldots, b_{n}[n]\right),
$$

for all $b_{1}, \ldots, b_{n} \in B$.

Fix some $c_{1} \in B_{1}, \ldots, c_{n} \in B_{n}$. The $n$-sorted algebra $\mathbf{B}_{*}$ will have the universe $\left(B_{1}, \ldots, B_{n}\right)$.

The interpretation of the $k$-ary function symbol $f \in \mathcal{L}$ of the type $\left\langle S_{i_{1}}, \ldots, S_{i_{k}}, S_{j}\right\rangle$ is defined in the following way: if $b_{1} \in B_{i_{1}}, \ldots, b_{k} \in B_{i_{k}}, f^{\mathbf{B}_{*}}\left(b_{1}, \ldots, b_{k}\right)$ is defined to be the $j$ th component of

$$
\begin{gathered}
\left(f^{*}\right)^{\mathbf{B}}\left(\left\langle c_{1}, \ldots, c_{i_{1}-1}, b_{1}, c_{i_{1}+1}, \ldots, c_{n}\right\rangle,\left\langle c_{1}, \ldots, c_{i_{2}-1}, b_{2}, c_{i_{2}+1}, \ldots, c_{n}\right\rangle,\right. \\
\left.\left\langle c_{1}, \ldots, c_{i_{k}-1}, b_{k}, c_{i_{k}+1}, \ldots, c_{n}\right\rangle\right) .
\end{gathered}
$$

It is readily seen that, for any algebra $\mathbf{A}$ of signature $\mathcal{L}$ and any algebra $\mathbf{B}$ of signature $\mathcal{L}^{*}$, satisfying $\Sigma$,

$$
\left(\mathbf{A}^{*}\right)_{*} \cong \mathbf{A},
$$

and

$$
\left(\mathbf{B}_{*}\right)^{*} \cong \mathbf{B} .
$$

For each term $t\left(x_{1}, \ldots, x_{k}\right)$ in $\mathcal{L}$, we define the term

$$
t^{*}\left(x_{1}, \ldots, x_{k}\right)
$$

in $\mathcal{L}^{*}$, as follows:

$1^{\circ}$ If $t\left(x_{1}, \ldots, x_{k}\right)$ is a variable $x_{i}$, then $t^{*}\left(x_{1}, \ldots, x_{k}\right)$ is an (unsorted) variable $x_{i}$.

$2^{\circ}$ If $t\left(x_{1}, \ldots, x_{k}\right)$ is of the form

$$
f\left(s_{1}\left(x_{1}, \ldots, x_{k}\right), \ldots, s_{l}\left(x_{1}, \ldots, x_{k}\right)\right),
$$

where $f \in \mathcal{L}$ is $l$-ary of type $\left\langle S_{i_{1}}, \ldots, S_{i_{l}}, S_{j}\right\rangle$, and $s_{i_{m}}\left(x_{1}, \ldots, x_{k}\right)$ is of the sort $S_{i_{m}}(1 \leqslant m \leqslant l)$, we define $t^{*}\left(x_{1}, \ldots, x_{k}\right)$ to be

$$
f^{*}\left(s_{1}^{*}\left(x_{1}, \ldots, x_{k}\right), \ldots, s_{l}^{*}\left(x_{1}, \ldots, x_{k}\right)\right)
$$

and all the variables $x_{1}, \ldots, x_{k}$ are unsorted.

If $\psi$ is an equation in $\mathcal{L}$ of the form

$$
t_{1}\left(x_{1}, \ldots, x_{k}\right)=t_{2}\left(x_{1}, \ldots, x_{k}\right),
$$

where $t_{1}, t_{2}$ are terms, which are both of the sort $S_{i}$, define $\psi^{*}$ to be the following equation in $\mathcal{L}^{*}$ :

$$
\begin{aligned}
& d\left(x_{1}, \ldots, x_{1}, t_{1}^{*}\left(x_{1}, \ldots, x_{k}\right), x_{1}, \ldots, x_{1}\right) \\
= & d\left(x_{1}, \ldots, x_{1}, t_{2}^{*}\left(x_{1}, \ldots, x_{k}\right), x_{1}, \ldots, x_{1}\right),
\end{aligned}
$$

where $t_{1}^{*}$ and $t_{2}^{*}$ occur as the $i$-th entries in the list of arguments of $d$. 
Given a formula $\phi$ in $\mathcal{L}$, we define $\phi^{*}$ to be a formula in $\mathcal{L}^{*}$ obtained by replacing every atomic subformula $\psi$ of $\phi$ by $\psi^{*}$ and making all variables unsorted.

Lemma 2.7. Let $\mathbf{A}$ be an algebra of signature $\mathcal{L}$, and let $a_{1}, \ldots, a_{k} \in A^{*}$, where

$$
a_{i}=\left\langle a_{1}^{i}, \ldots, a_{n}^{i}\right\rangle \in S_{1}^{\mathbf{A}} \times \cdots \times S_{n}^{\mathbf{A}}, \quad(1 \leqslant i \leqslant k) .
$$

If $t\left(x_{1}, \ldots, x_{k}\right)$ is a term of the type $\left\langle S_{i_{1}}, \ldots, S_{i_{k}}, S_{j}\right\rangle$ in $\mathcal{L}$, then in $\mathbf{A}^{*}$, the $j$ th component of

$$
\left(t^{*}\right)^{\mathbf{A}^{*}}\left(a_{1}, \ldots, a_{k}\right)
$$

is

$$
t^{\mathbf{A}}\left(a_{i_{1}}^{1}, \ldots, a_{i_{k}}^{k}\right)
$$

Proposition 2.8. Let $\mathbf{A}$ be an algebra of signature $\mathcal{L}$ and $\phi\left(x_{1}, \ldots, x_{k}\right)$ a formula in $\mathcal{L}$, whose variables are of the sorts $S_{i_{1}}, \ldots, S_{i_{k}}$, respectively. Let $a_{1} \in$ $S_{i_{1}}^{\mathbf{A}}, \ldots, a_{k} \in S_{i_{k}}^{\mathbf{A}}$, and let $b_{1}, \ldots, b_{k} \in A^{*}$ be such that

$$
b_{1}\left[i_{1}\right]=a_{1}, \ldots, b_{k}\left[i_{k}\right]=a_{k} .
$$

Then,

$$
\mathbf{A} \models \phi\left(a_{1}, \ldots, a_{k}\right) \quad \text { iff } \quad \mathbf{A}^{*} \models \phi^{*}\left(b_{1}, \ldots, b_{k}\right) .
$$

Proof. The proof is by induction on the complexity of $\phi$.

$1^{\circ} \phi$ is an equation of the form

$$
t_{1}\left(x_{1}, \ldots, x_{k}\right)=t_{2}\left(x_{1}, \ldots, x_{k}\right) .
$$

Then,

$$
\mathbf{A} \models t_{1}^{\mathbf{A}}\left(a_{1}, \ldots, a_{k}\right)=t_{2}^{\mathbf{A}}\left(a_{1}, \ldots, a_{k}\right)
$$

if and only if

$$
\begin{gathered}
\mathbf{A}^{*}=d^{\mathbf{A}^{*}}\left(b_{1}, \ldots, b_{1},\left(t_{1}^{*}\right)^{\mathbf{A}^{*}}\left(b_{1}, \ldots, b_{k}\right), b_{1}, \ldots, b_{1}\right) \\
=d^{\mathbf{A}^{*}}\left(b_{1}, \ldots, b_{1},\left(t_{2}^{*}\right)^{\mathbf{A}^{*}}\left(b_{1}, \ldots, b_{k}\right), b_{1}, \ldots, b_{1}\right),
\end{gathered}
$$

where $t_{1}^{*}, t_{2}^{*}$ occur as the $i$-th entries in the list of the arguments of $d$. (Here, we have made use of the Lemma [2.7) This is, in turn, equivalent to

$$
\mathbf{A}^{*} \models \phi^{*}\left(b_{1}, \ldots, b_{k}\right) .
$$

$2^{\circ}$ If $\phi\left(x_{1}, \ldots, x_{k}\right)$ is either $\neg \psi\left(x_{1}, \ldots, x_{k}\right)$ or $\psi\left(x_{1}, \ldots, x_{k}\right) \wedge \theta\left(x_{1}, \ldots, x_{k}\right)$, for some formulas $\psi\left(x_{1}, \ldots, x_{k}\right), \theta\left(x_{1}, \ldots, x_{k}\right)$, the claim follows immediately.

$3^{\circ}$ Assume that $\phi\left(x_{1}, \ldots, x_{k}\right)$ is of the form

$$
\left(\exists y \in S_{i}\right) \psi\left(y, x_{1}, \ldots, x_{k}\right) .
$$

Then,

$$
\mathbf{A} \models\left(\exists y \in S_{i}\right) \psi\left(y, a_{1}, \ldots, a_{k}\right)
$$

iff there exists $a \in S_{i}^{\mathbf{A}}$ such that

$$
\mathbf{A} \models \psi\left(a, a_{1}, \ldots, a_{k}\right) .
$$

Choosing $b \in A^{*}$ to be an arbitrary $n$-tuple whose $i$-th coordinate is $a$, and applying the induction hypothesis, we get

$$
\mathbf{A} \models \psi\left(a, a_{1}, \ldots, a_{k}\right) \quad \text { iff } \quad \mathbf{A}^{*} \models \psi^{*}\left(b, b_{1}, \ldots, b_{k}\right) .
$$


Since $\phi^{*}$ is

$$
\left(\exists y \in S_{i}\right) \psi\left(y, x_{1}, \ldots, x_{k}\right)
$$

this yields

$$
\mathbf{A} \models\left(\exists y \in S_{i}\right) \psi\left(y, a_{1}, \ldots, a_{k}\right) \quad \text { iff } \quad \mathbf{A}^{*} \models(\exists y) \psi^{*}\left(y, b_{1}, \ldots, b_{k}\right) .
$$

Corollary 2.9. If $\mathbf{A}$ is any algebra of signature $\mathcal{L}$, and $\phi$ a sentence in $\mathcal{L}$, then

$$
\mathbf{A} \models \phi \quad \text { iff } \quad \mathbf{A}^{*} \models \phi^{*} .
$$

Our next task is to define the corresponding effective transformation on sentences in $\mathcal{L}^{*}$. We first establish certain properties of terms in $\mathcal{L}^{*}$.

Lemma 2.10. Let $\mathbf{B}$ be an algebra of signature $\mathcal{L}^{*}$, satisfying $\Sigma$. If $t\left(x_{1}, \ldots, x_{k}\right)$ is a term in $\mathcal{L}$ of the type $\left\langle S_{i_{1}}, \ldots, S_{i_{k}}, S_{j}\right\rangle$, and $b_{1}, \ldots, b_{k} \in B$, then any coordinate of

$$
\left(t^{*}\right)^{\mathbf{B}}\left(b_{1}, \ldots, b_{k}\right)
$$

is equal to

$$
s^{\mathbf{B}_{*}}\left(b_{1}\left[i_{1}\right], \ldots, b_{k}\left[i_{k}\right]\right),
$$

for some subterm $s\left(x_{1}, \ldots, x_{k}\right)$ of $t$.

Proof. The proof is by induction on the complexity of $t$.

$1^{\circ} t\left(x_{1}, \ldots, x_{k}\right)$ is a variable $x_{m}(1 \leqslant m \leqslant k)$, of sort $S_{i_{m}}$. In this case, the claim is trivial.

$2^{\circ}$ Suppose $t\left(x_{1}, \ldots, x_{k}\right)$ is of the form

$$
f\left(s_{1}\left(x_{1}, \ldots, x_{k}\right), \ldots, s_{m}\left(x_{1}, \ldots, x_{k}\right)\right),
$$

where each $s_{r}\left(x_{1}, \ldots, x_{k}\right)$ is of the type $\left\langle S_{i_{1}}, \ldots, S_{i_{k}}, S_{j_{r}}\right\rangle(1 \leqslant r \leqslant m)$, while $f$ is of the type $\left\langle S_{j_{1}}, \ldots, S_{j_{r}}, S_{j}\right\rangle$. Then,

$$
\begin{aligned}
&\left(f^{*}\right)^{\mathbf{B}}\left(\left(s_{1}^{*}\right)^{\mathbf{B}}(\bar{b}), \ldots,\left(s_{m}^{*}\right)^{\mathbf{B}}(\bar{b})\right) \\
&=\left\langle\left(s_{1}^{*}\right)^{\mathbf{B}}(\bar{b})[1], \ldots,\left(s_{1}^{*}\right)^{\mathbf{B}}(\bar{b})[j-1], f^{\mathbf{B}_{*}}\left(\left(s_{1}^{*}\right)^{\mathbf{B}}(\bar{b})[i], \ldots,\left(s_{m}^{*}\right)^{\mathbf{B}}(\bar{b})[i]\right), \ldots,\right. \\
&\left.\left(s_{1}^{*}\right)^{\mathbf{B}}(\bar{b})[j+1], \ldots,\left(s_{1}^{*}\right)^{\mathbf{B}}(\bar{b})[n]\right\rangle,
\end{aligned}
$$

and the claim follows from the induction hypothesis applied to $s_{1}^{*}(\bar{b}), \ldots, s_{m}^{*}(\bar{b})$.

Moreover, observe that, from the proof of the lemma, we have the following: for each $t\left(x_{1}, \ldots, x_{k}\right)$ and $b_{1}, \ldots, b_{k} \in B$, we can effectively recover the subterms $s_{1}, \ldots, s_{n}$ of $t$, which determine the coordinates of $t^{*}\left(b_{1}, \ldots, b_{k}\right)$ in a unique way.

Lemma 2.11. Modulo $\Sigma$, every term $t^{*}\left(x_{1}, \ldots, x_{k}\right)$ in $\mathcal{L}^{*}$ is equal to a term of the form

$$
d\left(t_{1}^{*}\left(x_{1}, \ldots, x_{k}\right), \ldots, t_{n}^{*}\left(x_{1}, \ldots, x_{k}\right)\right),
$$

and $d$ does not occur in any of $t_{1}^{*}, \ldots, t_{n}^{*}$. 
Proof. The proof is by induction on the complexity of $t^{*}$.

$1^{\circ}$ If $t^{*}\left(x_{1}, \ldots, x_{k}\right)$ is $f^{*}\left(x_{1}, \ldots, x_{k}\right)$, then

$$
t^{*}\left(x_{1}, \ldots, x_{k}\right)=d\left(f^{*}\left(x_{1}, \ldots, x_{k}\right), \ldots, f^{*}\left(x_{1}, \ldots, x_{k}\right)\right) .
$$

If $t^{*}\left(x_{1}, \ldots, x_{k}\right)$ is $d\left(x_{i_{1}}, \ldots, x_{i_{n}}\right)$, where $\left\{x_{i_{1}}, \ldots, x_{i_{n}}\right\} \subseteq\left\{x_{1}, \ldots, x_{k}\right\}$, then $t^{*}$ itself will do.

$2^{\circ}$ If $t^{*}\left(x_{1}, \ldots, x_{k}\right)$ is of the form

$$
d\left(s_{1}^{*}\left(x_{1}, \ldots, x_{k}\right), \ldots, s_{n}^{*}\left(x_{1}, \ldots, x_{k}\right)\right),
$$

let

$$
s_{i}^{*}\left(x_{1}, \ldots, x_{k}\right)=d\left(t_{i, 1}^{*}\left(x_{1}, \ldots, x_{k}\right), \ldots, t_{i, n}^{*}\left(x_{1}, \ldots, x_{k}\right)\right),
$$

where none of $t_{i, 1}^{*}, \ldots, t_{n, i}^{*}$ contains $d$, for $1 \leqslant i \leqslant n$. Then,

$$
\begin{aligned}
t^{*}\left(x_{1}, \ldots, x_{k}\right) & =d\left(d\left(t_{1,1}^{*}(\bar{x}), \ldots, t_{1, n}^{*}(\bar{x})\right), \ldots, d\left(t_{n, 1}^{*}(\bar{x}), \ldots, t_{n, n}^{*}(\bar{x})\right)\right) \\
& =d\left(t_{1,1}^{*}(\bar{x}), t_{2,2}^{*}(\bar{x}), \ldots, t_{n, n}^{*}(\bar{x})\right) .
\end{aligned}
$$

If $t^{*}\left(x_{1}, \ldots, x_{k}\right)$ is of the form

$$
f^{*}\left(s_{1}(\bar{x}), \ldots, s_{k}(\bar{x})\right),
$$

where $f^{*}$ is $k$-ary, using the induction hypothesis, we get

$$
s_{i}^{*}(\bar{x})=d\left(t_{i, 1}^{*}(\bar{x}), \ldots, t_{i, n}^{*}(\bar{x})\right),
$$

where none of $t_{i, j}^{*}$ contains $d$. Then,

$$
\begin{gathered}
t^{*}(\bar{x})=f^{*}\left(d\left(t_{1,1}^{*}(\bar{x}), \ldots, t_{1, n}^{*}(\bar{x})\right), \ldots, d\left(t_{k, 1}^{*}(\bar{x}), \ldots, t_{k, n}^{*}(\bar{x})\right)\right) \\
\left.=d\left(t_{1,1}^{*}(\bar{x}), \ldots, t_{j-1,1}^{*}(\bar{x}), f\left(t_{1, i}^{*}(\bar{x}), \ldots, t_{k, i}^{*}(\bar{x})\right), t_{j+1,1}^{*}(\bar{x}), \ldots, t_{n, 1}^{*}(\bar{x})\right)\right) .
\end{gathered}
$$

This concludes the proof of the lemma.

Hence, we can effectively transform any equation $\psi$ in $\mathcal{L}^{*}$ into one of the form from the statement of Lemma 2.11. So, by Lemma 2.11, without any loss of generality, we may assume that any equation in $\mathcal{L}^{*}$ is of the form

$$
\begin{aligned}
& d\left(t_{1}^{*}\left(x_{1}, \ldots, x_{k}\right), \ldots, t_{n}^{*}\left(x_{1}, \ldots, x_{k}\right)\right) \\
= & d\left(u_{1}^{*}\left(x_{1}, \ldots, x_{k}\right), \ldots, u_{n}^{*}\left(x_{1}, \ldots, x_{k}\right)\right),
\end{aligned}
$$

where the terms $t_{i}^{*}, u_{j}^{*}$ contain no occurrences of $d$.

Given an equation $\psi$ in $\mathcal{L}^{*}$ of the form (2.6), define $\psi_{*}$ as follows:

$$
\psi_{*}=\bigwedge_{i=1}^{n} \theta_{i}
$$

where $\theta_{i}$ is constructed in the following way: let $p_{i}$ be the subterm of $t_{i}$, such that, for every $b_{1}, \ldots, b_{k} \in B$,

$$
t_{i}^{*}\left(b_{1}, \ldots, b_{k}\right)[i]=p_{i}\left(b_{1}\left[i_{1}\right], \ldots, b_{k}\left[i_{k}\right]\right),
$$

where $t_{i}$ is of the type $\left\langle S_{i_{1}}, \ldots, S_{i_{k}}, S_{j}\right\rangle$, and let $q_{i}$ be the subterm of $u_{i}$ with the same property, namely

$$
u_{i}^{*}\left(b_{1}, \ldots, b_{k}\right)[i]=q_{i}\left(b_{1}\left[j_{1}\right], \ldots, b_{k}\left[j_{k}\right]\right) .
$$

Set $\theta_{i}$ to be equal to

$$
p_{i}\left(x_{i_{1}}^{1}, \ldots, x_{i_{k}}^{k}\right)=q_{i}\left(x_{j_{1}}^{1}, \ldots, x_{j_{k}}^{k}\right) .
$$


Now, if $\phi\left(x_{1}, \ldots, x_{k}\right)$ is a formula in $\mathcal{L}^{*}$, let

$$
\phi_{*}\left(x_{1}^{1}, \ldots, x_{n}^{1}, x_{1}^{2}, \ldots, x_{n}^{2}, \ldots, x_{1}^{k}, \ldots, x_{n}^{k}\right)
$$

be the formula in $\mathcal{L}$, obtained by replacing all equations (atomic subformulas) $\psi$ of $\phi$ by $\psi_{*}$ as described above. Also, each quantifier $Q x_{i}$ in $\phi$ will be replaced by

$$
Q x_{1}^{i} \cdots x_{n}^{i} \text {. }
$$

Clearly, if $\phi$ contains $m$ variables, $\phi_{*}$ will contain $m \cdot n$ variables.

Proposition 2.12. Suppose

$$
\mathbf{B} \models \Sigma .
$$

For

$$
b_{1}=\left\langle b_{1}^{1}, \ldots, b_{n}^{1}\right\rangle, \ldots, b_{k}=\left\langle b_{1}^{k}, \ldots, b_{n}^{k}\right\rangle
$$

in $B$, and a formula $\phi\left(x_{1}, \ldots, x_{k}\right)$ in $\mathcal{L}^{*}$, we have

$$
\mathbf{B} \models \phi\left(b_{1}, \ldots, b_{k}\right) \quad \text { iff } \quad \mathbf{B}_{*}=\phi_{*}\left(b_{1}^{1}, \ldots, b_{n}^{1}, \ldots, b_{1}^{k}, \ldots, b_{n}^{k}\right) .
$$

Proof. If $\phi$ is an equation in $\mathcal{L}^{*}$, the validity of the claim follows from the preceding discussion. The only remaining case, which is nontrivial, is when $\phi\left(x_{1}, \ldots, x_{k}\right)$ is of the form

$$
(\exists x) \psi\left(x, x_{1}, \ldots, x_{k}\right)
$$

Then,

$$
\mathbf{B} \models(\exists x) \psi\left(x, b_{1}, \ldots, b_{k}\right)
$$

if and only if there exists

$$
b_{0}=\left\langle b_{1}^{0}, \ldots, b_{n}^{0}\right\rangle
$$

in $B$ such that

$$
\mathbf{B} \models \psi\left(b, b_{1}, \ldots, b_{k}\right) .
$$

Then, by the induction hypothesis,

$$
\mathbf{B} \models \psi\left(b, b_{1}, \ldots, b_{k}\right)
$$

if and only if

$$
\mathbf{B}_{*} \models \psi_{*}\left(b_{1}^{0}, \ldots, b_{n}^{0}, b_{1}^{1}, \ldots, b_{n}^{1}, \ldots, b_{1}^{k}, \ldots, b_{n}^{k}\right),
$$

but observe that

$$
\psi_{*}\left(x_{1}^{1}, \ldots, x_{n}^{1}, \ldots, x_{1}^{k}, \ldots, x_{n}^{k}\right)
$$

is actually

$$
\left(\exists x_{1} \cdots x_{n}\right) \psi_{*}\left(x_{1}, \ldots, x_{n}, x_{1}^{1}, \ldots, x_{n}^{1}, \ldots, x_{1}^{k}, \ldots, x_{n}^{k}\right) .
$$

An immediate corollary of Proposition 2.12 is:

Corollary 2.13. For any algebra $\mathbf{B}$ of signature $\mathcal{L}^{*}$ and any sentence $\phi$ in $\mathcal{L}^{*}$,

$$
\mathbf{B} \models \phi \quad \text { iff } \quad \mathbf{B}_{*} \models \phi_{*} .
$$


2.4. Adding the discriminator. Let $\mathcal{K}$ be a class of algebras of a 1-sorted signature $\mathcal{L}$, axiomatized by a finite set of universal sentences $\Phi$ in $\mathcal{L}$, which is universally pseudorecursive.

Definition 2.14. Let $A$ be a nonempty set. The ternary discriminator function $t$ on $A$ is defined by

$$
t(a, b, c)= \begin{cases}a, & \text { if } a \neq b, \\ c, & \text { if } a=b\end{cases}
$$

for $a, b, c \in A$.

Let $\mathcal{K}^{t}$ be a class of algebras of signature $\mathcal{L} \cup\{t\}$, where $t$ is a ternary function symbol not occurring in $\mathcal{L}$, and which is axiomatized by $\Phi \cup\left\{\delta_{t}\right\}$, where $\delta_{t}$ is a universal sentence asserting that $t$ is interpreted by the discriminator function in every algebra in $\mathcal{K}^{t}$. Also, for any algebra $\mathbf{A} \in \mathcal{K}$, let $\mathbf{A}^{t}$ stand for the expansion of $\mathbf{A}$ by the discriminator function on $A$.

Clearly, we have the following:

Proposition 2.15. $\mathcal{K}^{t}$ has the undecidable universal theory.

Now, given a universal sentence $\phi$ in $\mathcal{L}^{t}=\mathcal{L} \cup\{t\}$, one can effectively construct a universal sentence $\phi_{*}$ in $\mathcal{L}$ with the same number of variables, so that

$$
\mathbf{A} \models \phi_{*} \quad \text { iff } \quad \mathbf{A}^{t} \models \phi,
$$

where $\mathbf{A}$ is any algebra in $\mathcal{K}$.

Here is how the construction proceeds. If $\phi$ contains an atomic subformula of the form

$$
t\left(s_{1}, s_{2}, s_{3}\right)=s,
$$

where $s_{1}, s_{2}, s_{3}, s$ are terms in $\mathcal{L}^{t}$, replace it by

$$
\left(s_{1}=s_{2} \wedge s_{3}=s\right) \vee\left(s_{1} \neq s_{2} \wedge s_{1}=s\right) .
$$

Since $t\left(s_{1}, s_{2}, s_{3}\right)=s$ is equivalent to (2.7) in any model of $\Phi \cup\left\{\delta_{t}\right\}$, eliminating $t$ successively in $\phi$, in the manner described above, one obtains $\phi_{*}$ such that

$$
\mathbf{A}^{t}=\phi \quad \text { iff } \quad \mathbf{A}=\phi_{*} .
$$

Now, it is easy to prove the following:

Proposition 2.16. $T h_{\forall, n}\left(\mathcal{K}^{t}\right)$ is decidable, for every $n<\omega$.

Consider the variety $\mathbf{V}\left(\mathcal{K}^{t}\right)$. A result from [10] shows that, for each universal sentence $\phi$ in $\mathcal{L}^{t}$, one can effectively construct an identity $\hat{\phi}$ in $\mathcal{L}^{t}$ such that, in every subdirectly irreducible algebra $\mathbf{A}$ in $\mathbf{V}\left(\mathcal{K}^{t}\right)$,

$$
\mathbf{A} \models \phi \quad \text { iff } \quad \mathbf{A} \models \hat{\phi} .
$$

But $\mathcal{K}^{t}$ consists of the subdirectly irreducible algebras in $\mathbf{V}\left(\mathcal{K}^{t}\right)$ plus the oneelement algebras (in case $\mathcal{K}$ has any one-element algebras). Therefore, an equation is true in $\mathbf{V}\left(\mathcal{K}^{t}\right)$ if and only if it is true in $\mathcal{K}^{t}$. Hence, the undecidability of the universal theory of $\mathcal{K}^{t}$ implies the undecidability of the equational theory of $\mathbf{V}\left(\mathcal{K}^{t}\right)$.

Theorem 2.17. $\mathbf{V}\left(\mathcal{K}^{t}\right)$ has the undecidable equational theory. 
Another result from [10] shows that $\mathbf{V}\left(\mathcal{K}^{t}\right)$ is finitely based. Namely, the axiomatization of $\mathbf{V}\left(\mathcal{K}^{t}\right)$ is given by the equational translations of universal sentences in $\Phi \cup\left\{\delta_{t}\right\}$ plus the identities relating the discriminator to other symbols in $\mathcal{L}$.

Since $T h_{\forall, n}\left(\mathcal{K}^{t}\right)$ is decidable, for every $n<\omega, T h_{Q, n}\left(\mathcal{K}^{t}\right)$, the theory of quasiidentities of $\mathcal{K}^{t}$ with at most $n$ variables, is decidable.

It is a well-known fact that the $T h_{Q}\left(\mathcal{K}^{t}\right)$ is precisely $T h_{Q}\left(\mathbf{V}\left(\mathcal{K}^{t}\right)\right)$, whence $T h_{Q, n}\left(\mathbf{V}\left(\mathcal{K}^{t}\right)\right)$ is decidable, for every $n<\omega$.

Yet another well-known fact is that the decidability of $T h_{Q, n}\left(\mathbf{V}\left(\mathcal{K}^{t}\right)\right)$ implies the existence of a uniform algorithm, which solves the word problem for any finitely presented algebra in $\mathbf{V}\left(\mathcal{K}^{t}\right)$ with at most $n$ generators in its finite presentation. This yields the following theorem:

Theorem 2.18. Every finitely presented algebra in $\mathbf{V}\left(\mathcal{K}^{t}\right)$ has solvable word problems.

By combining all the steps so far, we complete the proof of Theorem 1.2 .

\section{Definition of the Class $\mathcal{K}$}

In this section, our goal is to construct a class $\mathcal{K}$ of structures in some multisorted language $\mathcal{L}$, axiomatized by a finite set of universal sentences in $\mathcal{L}$, which will have the desired properties; namely, its universal theory will be undecidable, while the theory consisting of the universal sentences true in $\mathcal{K}$ involving at most $n$ variables of each sort will be decidable, for every $n \in \omega$.

To ensure that the universal theory of $\mathcal{K}$ is undecidable, we design $\mathcal{K}$ so as to reflect the computations of a Turing machine with the undecidable Halting problem.

On the other hand, the decidability of every $(n, n, \ldots, n)$-universal theory will follow from the fact that, given a universal sentence $\phi$ in $\mathcal{L}$ containing at most $n$ variables of each sort, we can effectively check whether $\phi$ is false in some structure in $\mathcal{K}$, by listing effectively all structures in $\mathcal{K}$ generated by at most $n$ elements of each sort and $(n, n, \ldots, n)$-elements belonging to them.

Namely, if a structure is generated by at most $n$ elements of every sort, there will be only finitely many finite components (which will be defined later) in such a structure, and that number will be bounded by some computable function of $n$. These finite components, together with their location in the structure, and at most $n$ configurations of our Turing machine will, speaking informally, completely determine the structure.

The version of a Turing machine that we use is such that the tape of $T$, is semiinfinite (to the right), and, if it happens that the machine head is scanning the leftmost cell of the tape and the appropriate instruction directs it to the left, the machine halts in no state.

Throughout the rest of the paper, the machine $T$ in question is assumed to be such that its halting problem in state $q_{0}$ is undecidable. The version of the Halting problem that we have in mind, as mentioned in the introduction, is the following: there is a machine $T$ for which the set of the initial configurations which lead to the halting state is undecidable.

By a Turing machine we mean a quintuple

$$
T=\left\langle Q, S, \delta, q_{0}, q_{1}\right\rangle,
$$


where

- $S=\left\{s_{0}, s_{1}, \ldots, s_{m}, B\right\}$ is the set of the tape symbols of $T$, where $B$ stands for the blank symbol;

- $Q=\left\{q_{0}, q_{1}, \ldots, q_{n}\right\}$ is the set of the states of $T$;

- $q_{0} \in Q$ is the unique halting state of $T$;

- $q_{1} \in Q$ is the initial state of $T$;

- $\delta: S \times Q \rightarrow S \times Q \times\{L, N, R\}$ is a partial mapping (the transition function of $T$ ) and the intended interpretation of

$$
\delta(s, q)=\left(s^{\prime}, q^{\prime}, \Gamma\right), \quad \Gamma \in\{L, N, R\}
$$

is: $T$, upon reading $s$ in the scanned cell, with its head in the state $q$, prints $s^{\prime}$ in place of $s$, moves in the direction $\Gamma$ ( $L$-left, $N$-no move, $R$-right) changing the state of its head to $q^{\prime}$.

Also, the transition function $\delta$ of $T$ is assumed to satisfy the following: for every $s \in S$

$$
\delta\left(s, q_{0}\right)=\left(s, q_{0}, N\right) .
$$

I.e., once the halting state has been reached there is no further action of $T$.

By a configuration we mean a finite initial portion of the tape, together with the current position of the head and its current state (we also allow for the case where the head is off the tape); i.e. a configuration is a finite sequence from $S \cup(S \times Q)$, having at most one coordinate from $S \times Q$. The set of all configurations of $T$ will be denoted by Config.

$T$ determines a unary function $T:$ Config $\rightarrow$ Config via its transition function $\delta$. For practical purposes, we shall assume that $T$, acting on Config, always increases the length of the configuration by 1 , by, if necessary, adding a blank symbol to the right.

If $C \in$ Config is of the form

$$
C=\left(s_{i_{1}}, q\right) s_{i_{2}} \cdots s_{i_{n}}
$$

and

$$
\delta\left(s_{i_{1}}, q\right)=\left(s^{\prime}, q^{\prime}, L\right)
$$

then

$$
T(C)=s^{\prime} s_{i_{2}} \cdots s_{i_{n}} B
$$

Before proceeding to the axiomatization of $\mathcal{K}$, we sketch the idea of a computation structure. These structures will be in $\mathcal{K}$. They are intended to fully capture computations of $T$. Our axiomatization for $\mathcal{K}$ results from assembling finitely many universal sentences which are true in these structures and which describe how these structures capture computations.

Namely, given a configuration $C$ of $T$, we can associate to it the computation structure $\mathbf{S}_{C}$. See Diagram 1.

Each horizontal line captures a particular configuration from the computation of $T$ when started on $C$. The computation proceeds upwards, one line at a time, in the following manner: assume that, given a configuration

$$
C_{1}: \quad s_{i_{1}} s_{i_{2}} \cdots\left(s_{i_{j}}, q\right) \cdots s_{i_{n}},
$$

$T\left(C_{1}\right)=C_{2}$, where $C_{2}$ is, say,

$$
C_{2}: \quad s_{i_{1}} s_{i_{2}} \cdots\left(s_{i_{j-1}}, q^{\prime}\right) s_{i_{j}}^{\prime} \cdots s_{i_{n}} B
$$




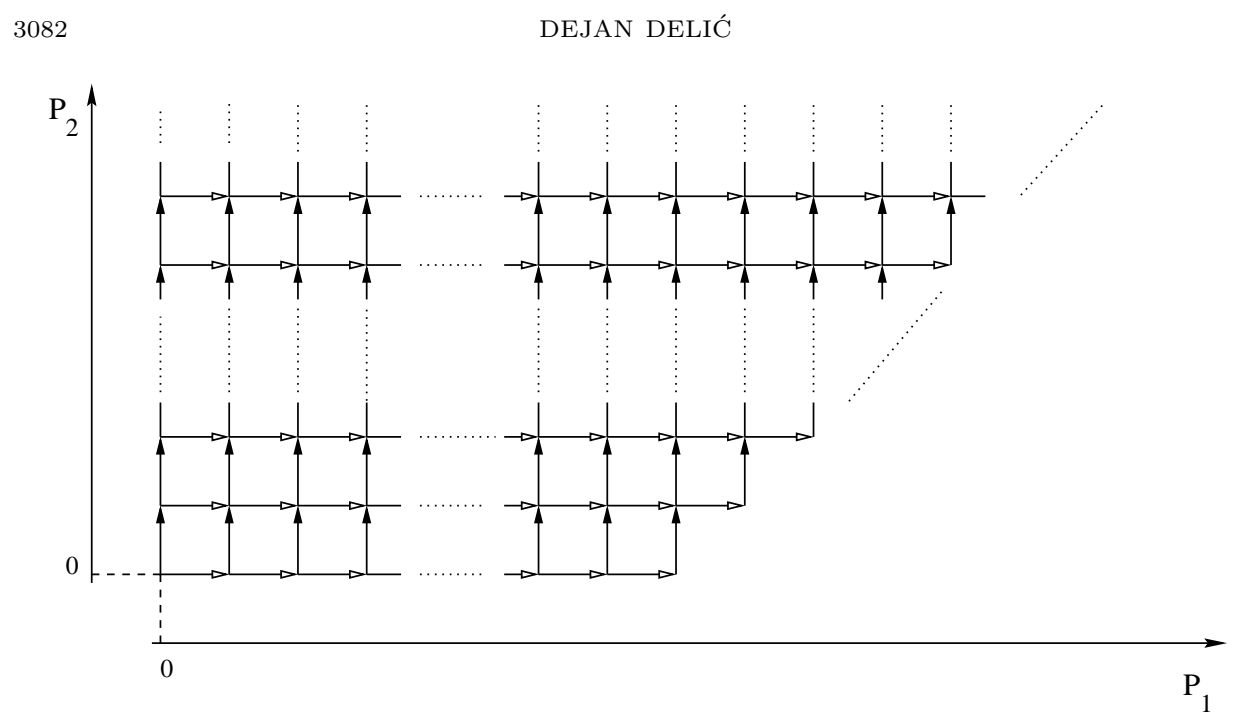

DiAGRAM 1.

$C_{2}$ will constitute the next "level" of our structure, being immediately "above" $C_{1}$. In other words, we introduce a new relation $\rightarrow$ which will connect the encodings of $C_{1}$ and $C_{2}$ in the following way:

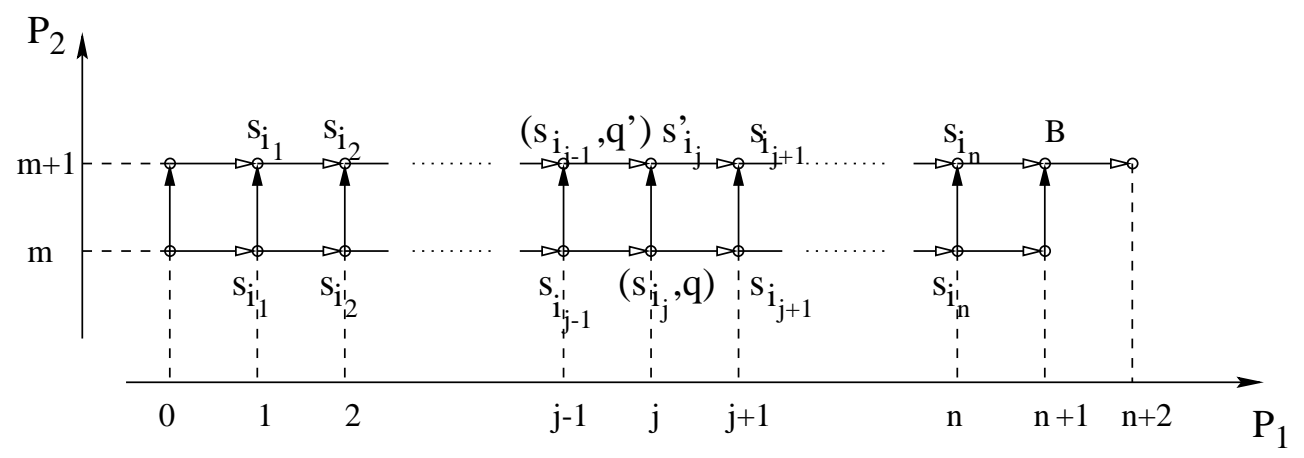

\section{DiAGRAM 2.}

The language $\mathcal{L}$ in which configuration structures, as well as the rest of the class $\mathcal{K}$, will be defined, has four sorts that will be denoted by $A, P_{1}, P_{2}$, and $C$. The symbols of $\mathcal{L}$ will be the following:

- Function symbols

$$
\begin{array}{ll}
\operatorname{diam}: A^{2} \rightarrow A & \text { bord }: A^{2} \rightarrow A \\
h: A^{3} \rightarrow A & \pi_{1}: A \rightarrow P_{1} \\
f: A^{4} \rightarrow A & \pi_{2}: A \rightarrow P_{2} \\
g: A^{2} \rightarrow A & \chi: A \rightarrow C
\end{array}
$$

- Relation symbols

$$
\begin{array}{ll}
\rightarrow \subseteq A \times A & \leqslant 1 \subseteq P_{1} \times P_{1} \\
\rightarrow \subseteq A \times A & \leqslant{ }_{2} \subseteq P_{2} \times P_{2}
\end{array}
$$


- Constant symbols

$$
\begin{array}{ll}
\infty \text { in } A & s \in S \text { in } C \\
\infty_{1} \text { in } P_{1} & (s, q) \in S \times Q \text { in } C \\
\infty_{2} \text { in } P_{2} & c \text { in } C \\
\infty^{\prime} \text { in } C & d \text { in } C \\
\diamond \text { in } C & \varnothing \text { in } C
\end{array}
$$

The computation structure is intended to correspond to a two-dimensional pattern in the grid that is coordinatized by two linear orderings, $\left(P_{1}, \leqslant_{1}\right)$ and $\left(P_{2}, \leqslant_{2}\right)$. $\pi_{1}$ and $\pi_{2}$ represent the projections of the "main structure part" $A$ on the coordinate axes $P_{1}$ and $P_{2}$. The role of $f, g, h$,diam and bord is to build $A$ according to the sequence of computations of $T$, started on $C$. If, at some point, a pattern is detected which cannot be a part of a genuine configuration of $T$, the appropriate structure-building operation has the default value $\infty . \infty_{1}$ and $\infty_{2}$ are the projections of $\infty$ onto $P_{1}$ and $P_{2}$, respectively. Finally, the role of the constant symbols $c$ and $d$ is to detect whether $T$ eventually halts; if at some point, the halting state $q_{0}$ is reached, the axioms force $c=d$ in $\mathbf{S}_{C}$.

The role of $\chi$ is to assign a "label" from $S \cup(S \times Q)$ to each element of $A$, so that this label indicates the tape/state symbol of $T$ at the corresponding position on the tape.

Definition 3.1. A computation structure $\mathbf{S}_{C}$, where $C$ is the configuration

$$
s_{1} s_{2} \ldots\left(s_{i}, q\right) \ldots s_{n}
$$

is a structure in the language $\mathcal{L}$ which is generated by elements $a_{0}, a_{1}, \ldots, a_{n}$, $a_{n+1} \in A$ such that

$$
\begin{gathered}
\chi\left(a_{0}\right)=\diamond, \chi\left(a_{1}\right)=s_{1}, \ldots, \chi\left(a_{i}\right)=\left(s_{i}, q\right), \ldots, \chi\left(a_{n}\right)=s_{n}, \chi\left(a_{n}+1\right)=\varnothing, \\
a_{0} \rightarrow a_{1} \rightarrow \ldots \rightarrow a_{n} \rightarrow a_{n+1}
\end{gathered}
$$

(similarly for the case where $C$ contains no elements of $S \times Q$ ).

We adopt the following convention: given a structure $\mathbf{S} \in \mathcal{K}$, the sets of elements of $\mathbf{S}$ of corresponding sorts, will be denoted by $A^{\mathbf{S}}, P_{1}^{\mathbf{S}}, P_{2}^{\mathbf{S}}, C^{\mathbf{S}}$, and

$$
\mathbf{S}=\left\langle A^{\mathbf{S}}, P_{1}^{\mathbf{S}}, P_{2}^{\mathbf{S}}, C^{\mathbf{S}}\right\rangle \text {. }
$$

The class $\mathcal{K}$ will be axiomatized by a finite list of universal sentences $\Phi$ in the language $\mathcal{L}$, whose explicit statements will be deferred to the appendix. These axioms ensure that all members of $\mathcal{K}$ share certain simple properties with the structures actually arising from computations of the Turing machine $T$. For example, some of the asserted properties of $\mathcal{K}$ are the following:

1. Every element of $A$ is labelled, via $\chi$, by some element of $S \cup(S \times Q) \cup$ $\left\{\diamond, \varnothing, \infty^{\prime}\right\}$, so that the first element of each line is labelled by $\diamond$, and the last one by $\varnothing$.

2. $\leqslant_{i}$ linearly orders $P_{i}$, with the largest element $\infty_{i}$.

3. The structure-building operations $f, g$, and $h$ reflect the computations of $T$. If the portion of a horizontal line of the computation structure looks like a genuine portion of the tape of $T$, the operations emulate the action of $T$ on that piece of the tape one level upwards; if this is not the case, the operations output the default element $\infty$.

4. diam puts $\diamond$ to the first position of the line representing a configuration, while bord marks the right end of a configuration by putting $\nabla$. 
5. $\rightarrow$ connects vertically the lines arising from two successive configurations in the computation of $T$, so that the elements labelled with $\diamond$ are vertically aligned, while there is no $x \in A$ which is $\rightarrow$-connected to an element labelled by $\varnothing$.

6. $\infty$ is isolated: i.e, there is no $x \in A$ such that either $x \rightarrow \infty$ or $x \rightarrow \infty$.

7. For every $s \in S$, there is an axiom which detects whether the final state $q_{0}$ has been reached:

$$
(\forall x \in A)\left(\chi(x)=\left(s, q_{0}\right) \Rightarrow c=d\right) .
$$

\section{Some important Features of $\mathcal{K}$}

In this section we investigate the structure of finitely generated models in the class $\mathcal{K}$, which was axiomatized by the set $\Phi$ of universal sentences in the previous section.

We assume that $\mathbf{S}=\left\langle A^{\mathbf{S}}, P_{1}^{\mathbf{S}}, P_{2}^{\mathbf{S}}, C^{\mathbf{S}}\right\rangle$ is a finitely generated member of $\mathcal{K}$, for which $A^{\mathbf{S}}$ is nonempty.

Let

$$
\mathbf{S}=S g\left(X, Y_{1}, Y_{2}, Z\right),
$$

where $X \neq \emptyset$, and $X, Y_{1}, Y_{2}$, and $Z$ are all finite. Also, define $\preccurlyeq$ to be the reflexivetransitive closure of $\rightarrow \mathbf{S} \cup \rightarrow^{\mathbf{S}}$ in $A^{\mathrm{S}}$.

The following lemma, whose proof will be omitted, follows essentially from the very definition of the class $\mathcal{K}$.

Lemma 4.1. If $\mathbf{S} \in \mathcal{K}$ is such that $X=\left\{a_{0}, \ldots, a_{m}\right\}$, where

$$
\begin{gathered}
a_{0} \rightarrow a_{1} \rightarrow \ldots \rightarrow a_{m-1} \rightarrow a_{m}, \\
\chi^{\mathbf{S}}\left(a_{0}\right)=\diamond, \chi^{\mathbf{S}}\left(a_{m}\right)=\varnothing, \\
\chi^{\mathbf{S}}\left(a_{i}\right) \in S \cup(S \times Q), 1 \leqslant i \leqslant m-1,
\end{gathered}
$$

so that for at most one $i$ such that $1 \leqslant i \leqslant m-1$,

$$
\chi^{\mathbf{S}}\left(a_{i}\right) \in S \times Q,
$$

then, for $\mathbf{S}_{\mathbf{1}}$, where $\mathbf{S}_{1}$ is generated by $X, \leqslant \mathbf{S}, \mathbf{S}_{\mathbf{1}}$ is a computation structure in $\mathcal{K}$.

Definition 4.2. Let $C$ be an intial configuration of the Turing machine $T$. We define $\theta_{C}$, the halting sentence corresponding to $C$, to be

$$
\begin{gathered}
\left(\forall x_{0} x_{1} \cdots x_{n+1} \in A\right)\left(x_{0} \rightarrow x_{1} \rightarrow \cdots \rightarrow x_{n} \rightarrow x_{n+1}\right. \\
\left.\wedge \chi\left(x_{0}\right)=\diamond \wedge \chi\left(x_{1}\right)=\left(s_{i_{1}}, q_{1}\right) \wedge \cdots \wedge \chi\left(x_{n}\right)=s_{i_{n}} \wedge \chi\left(x_{n+1}\right)=\varnothing \Rightarrow c=d\right) .
\end{gathered}
$$

Proposition 4.3. For any initial configuration $C$ of $T, \theta_{C} \in T h_{\forall}(\mathcal{K})$ if and only if $T$ halts when started on the configuration $C$.

Proof. Suppose the halting sentence is in the theory. Let $\mathbf{S}$ be the computation structure for $C$. Clearly, the elements corresponding to $C$ in $\mathbf{S}$ will fulfill the hypotheses of $\theta_{C}$. Thus,

$$
\mathbf{S} \models c=d .
$$

Therefore, $T$ halts when started on $C$.

Conversely, suppose $\theta_{C} \notin T h_{\forall}(\mathcal{K})$. Then, there exists a structure $\mathbf{S} \in \mathcal{K}$ and elements in $S$, which witness the failure of $\theta_{C}$ in $\mathbf{S}$. These elements will fulfill all the hypotheses of $\theta_{C}$, yet $c \neq d$ in $\mathbf{S}$. The selected elements will constitute a 
configuration line, and according to Lemma 4.1, the substructure $\mathbf{S}_{\mathbf{1}}$ of $\mathbf{S}$ generated by this line will be a configuration structure, in fact, the one which corresponds to $C$. Since $c \neq d$ in $\mathbf{S}_{\mathbf{1}}$, no element from $A^{\mathbf{S}_{1}}$ can be labelled with $\left(s, q_{0}\right)$ by $\chi$. Hence, $T$ does not halt when started on $C$.

As an immediate consequence of Proposition 4.3, we obtain

Theorem 4.4. $T h_{\forall}(\mathcal{K})$ is undecidable.

Our next task is to show that $T h_{\forall, n}(\mathcal{K})$ is decidable, for every $n<\omega$. In view of the Completeness Theorem, since $\mathcal{K}$ is finitely axiomatizable, it follows that $T h_{\forall, n}(\mathcal{K})$ is recursively enumerable, for every $n<\omega$. Thus, it remains to produce a recursive enumeration of the universal sentences with at most $n$ variables of each sort that are false in $\mathcal{K}$.

To do that, we construct an algorithm which lists finite fragments of the structures in $\mathcal{K}$ that are generated in each sort by at most $n$ elements. This will suffice since, as we show in the forthcoming series of arguments, in order to check for the failure of a given sentence from $T h_{\forall, n}(\mathcal{K})$ in such a structure, one only needs to recover a finite, "sufficiently large" portion of the structure.

We start with a series of lemmas about structural properties of such an $\mathbf{S}$.

Lemma 4.5. For every $a \in A^{\mathbf{S}} \backslash\left\{\infty^{\mathbf{S}}\right\}$, there exists $x \in X$ such that $x \preccurlyeq a$.

Proof. For an arbitrary subset $B \subseteq A^{\mathbf{S}}$, define

$$
\begin{aligned}
E(B)=\left(\left\{y \in A^{\mathbf{S}}: y=F^{\mathbf{S}}\left(x_{1}, \ldots,\right.\right.\right. & \left.x_{n}\right), \text { for some } x_{1}, \ldots, x_{n} \in B, \\
& \text { and some } \left.\left.F: A^{n} \rightarrow A \text { in } \mathcal{L}\right\} \cup B\right) \backslash\left\{\infty^{\mathbf{S}}\right\} .
\end{aligned}
$$

Now, let

$$
E^{(0)}(X)=X, \quad \text { and } \quad E^{(n+1)}(X)=E\left(E^{(n)}(X)\right), \quad \text { for } n<\omega .
$$

It is easily seen that, for $i \leqslant j$,

$$
E^{(i)}(X) \subseteq E^{(j)}(X)
$$

and

$$
A^{\mathbf{S}} \backslash\left\{\infty^{\mathbf{S}}\right\}=\bigcup_{n<\omega} E^{(n)}(X) .
$$

We prove the lemma by induction on $n$ such that $a \in E^{(n)}(X)$.

If $n=0$, then $a \in E^{(0)}(X)=X$, so $x=a$ would do.

Suppose that the lemma is true for every $n<k$ and let

$$
a \in E^{(k)}(X) \text {. }
$$

Then, either $a \in E^{(k-1)}(X)$ and we are done, or $a=F^{\mathbf{S}}\left(b_{1}, \ldots, b_{n}\right)$, for some $F: A^{n} \rightarrow A$ and $b_{1}, \ldots, b_{n} \in E^{(k-1)}$, so that, for some $i, i \in\{1, \ldots, n\} b_{i} \rightarrow a$, or, in case when $a=\operatorname{bord}\left(b_{1}, b_{2}\right)$, for some $b_{1}, b_{2} \in E^{(k-1)}(X)$,

$$
b_{2} \rightarrow b_{1} \rightarrow a \text {. }
$$

In any case, the induction hypothesis yields

$$
x \preccurlyeq a
$$

for some $x \in X$. 
Lemma 4.6. For every $b \in P_{i}^{\mathbf{S}},(i=1,2)$, either $b \in Y_{i}$ or $b=\pi_{i}(x)$, for some $x \in A^{\mathrm{S}}$.

Proof. Immediate, from the fact that the only operation which involves elements from $P_{i}^{\mathbf{S}}$ is $\pi_{i}^{\mathbf{S}}: A^{\mathbf{S}} \rightarrow P_{i}^{\mathbf{S}}$.

Let $X=\left\{x_{1}, \ldots, x_{n}\right\}$. For $1 \leqslant i \leqslant n$ we define

$$
B_{j}=\left\{a \in A^{\mathbf{S}}: x_{j} \preccurlyeq a\right\} .
$$

Then, from Lemma 4.5, we conclude that

$$
A^{\mathbf{S}} \backslash\left\{\infty^{\mathbf{S}}\right\}=B_{1} \cup \cdots \cup B_{n} .
$$

Lemma 4.7. For every $j \in\{1, \ldots, n\}$,

$$
\pi_{i}^{\mathbf{S}}\left(B_{j}\right)=\left\{\pi_{i}^{\mathbf{S}}(a): a \in B_{j}\right\}
$$

is well-ordered by $\leqslant_{i}$, for $i=1,2$.

Proof. If $x, y \in P_{i}^{\mathbf{S}}, x \neq y, x \leqslant i y$ and there is no $z \in P_{i}^{\mathbf{S}}, z \neq x, z \neq y$ such that

$$
x \leqslant{ }_{i} z \leqslant_{i} y,
$$

we write

$$
x \prec_{i} y .
$$

We prove the lemma for the case $i=1$, the proof for $i=2$ being similar.

Let $y \in \pi_{1}^{\mathbf{S}}\left(B_{j}\right)$ be arbitrary. Then,

$$
y=\pi_{1}^{\mathbf{S}}(a)
$$

for some $a \in A^{\mathrm{S}}$ such that $x_{j} \preccurlyeq a$. Let

$$
x_{j}=b_{0} \hookrightarrow_{1} b_{1} \hookrightarrow_{2} \cdots \hookrightarrow_{n-1} b_{m-1} \hookrightarrow_{n} b_{m}=a,
$$

where $\hookrightarrow_{1}, \ldots, \hookrightarrow_{n} \in\left\{\rightarrow_{\mathbf{S}_{\rightarrow}} \mathbf{S}\right\}, b_{1}, \ldots, b_{m-1} \in A^{\mathbf{S}}$. We can also assume that $b_{0}, b_{1}, \ldots, b_{m}$ are all distinct.

Let $\left\{c_{1}, \ldots, c_{l-1}\right\}=\left\{\pi_{1}^{\mathbf{S}}\left(b_{i}\right): 1 \leqslant i \leqslant m-1\right\}$, so that

$$
c_{1} \leqslant 1 c_{2} \leqslant 1 \ldots \leqslant 1 c_{l-1} .
$$

If $\hookrightarrow_{k}=\rightarrow \mathbf{S}$, then

$$
\pi_{1}^{\mathbf{S}}\left(b_{k-1}\right) \prec_{1} \pi_{1}^{\mathbf{S}}\left(b_{k}\right) .
$$

Hence,

$$
\pi_{1}^{\mathbf{S}}\left(x_{j}\right) \prec_{1} c_{1} \prec_{1} \cdots \prec_{1} c_{l-1} \prec_{1} y .
$$

Also, $\pi_{1}^{\mathbf{S}}\left(B_{j}\right)$ is at most countable, since $A^{\mathbf{S}}$ is such. Thus, $\pi_{1}^{\mathbf{S}}\left(B_{j}\right)$ is either a finite or countable well-ordering, isomorphic to $(\omega, \leqslant)$.

Proposition 4.8. $\left(P_{i}^{\mathbf{S}}, \leqslant_{i}\right)$ is a well-ordering, for $i=1,2$.

Proof. If $\mathbf{S}=S g\left(X, Y_{1}, Y_{2}, Z\right)$, due to Lemma 4.6,

$$
P_{i}^{\mathbf{S}}=Y_{i} \cup \pi_{I}^{\mathbf{S}}\left(B_{1}\right) \cup \cdots \cup \pi_{i}^{\mathbf{S}}\left(B_{n}\right) \cup\left\{\infty_{i}^{\mathbf{S}}\right\} .
$$

Clearly, each of the sets in this finite union is well-ordered, with respect to the restriction of $\leqslant_{i}, Y_{i}$ and $\left\{\infty_{i}^{\mathbf{S}}\right\}$ being finite, and $\pi_{i}^{\mathbf{S}}\left(B_{j}\right)$ by Lemma 1.7 .

Since every linearly ordered set that is a finite union of well-ordered sets is itself well-ordered, the proposition is proved. 
Thus,

$$
\left(P_{i}^{\mathbf{S}}, \leqslant_{i}\right) \cong\left(\lambda_{i}, \leqslant\right)
$$

where $\lambda_{i}$ is some, at most countable, ordinal which is yet to be determined.

From now on, we shall assume that

$$
P_{i}^{\mathbf{S}}=\lambda_{i}, \quad i=1,2 .
$$

A (horizontal) line in $A^{\mathrm{S}}$ is the set of all elements with the same $P_{2}$-coordinate; i.e. if $m \in \lambda_{2}$, the $m$-th horizontal line in $A^{\mathbf{S}}$ is

$$
l_{m}=\left\{x \in A^{\mathbf{S}}: \pi_{2}^{\mathbf{S}}(x)=m\right\} .
$$

Definition 4.9. If $l$ is a horizontal line in $A^{\mathrm{S}}$, then its index is an ordinal $m<\lambda_{2}$ such that

$$
l=l_{m}=\left\{x \in A^{\mathrm{S}}: \pi_{2}(x)=m\right\} .
$$

Lemma 4.10. Every horizontal line in $A^{\mathrm{S}}$ is finite.

Proof. We use the transfinite induction on the index of a line.

$1^{\circ}$ If the index of a line is a limit ordinal, then $l_{0} \subseteq X$, since there is neither an $a \in A^{\mathrm{S}}$ such that

$$
a \rightarrow x,
$$

nor $b, c \in A^{\mathrm{S}}$ such that

$$
b \rightarrow c \rightarrow x .
$$

Since $X$ is finite, then so is $l_{0}$.

$2^{\circ}$ Now, assume that $\mu$ is a successor ordinal in $\lambda_{2}, \mu=\nu+1$, and consider $l_{\mu}$. Every $x \in l_{\mu}$ is either a generator in $X$ or $F^{\mathbf{S}}\left(a_{1}, \ldots, a_{n}\right)$, for some $F: A^{n} \rightarrow A$, and $a_{1}, \ldots, a_{n} \in l_{\nu}$. Since $l_{\nu}$ is finite (by the induction hypothesis) and $\mathcal{L}$ is such, $l_{\mu}$ is finite as well.

Definition 4.11. A configuration line in $A^{\mathrm{S}}$ is a line consisting of $a_{0}, a_{1}, \ldots$, $a_{m}, a_{m+1} \in A^{\mathrm{S}}$, such that

$$
\begin{gathered}
a_{0} \rightarrow a_{1} \rightarrow \cdots \rightarrow a_{m} \rightarrow a_{m+1}, \\
\chi^{\mathbf{S}}\left(a_{0}\right)=\diamond, \chi^{\mathbf{S}}\left(a_{m+1}\right)=\varnothing, \\
\chi^{\mathbf{S}}\left(a_{i}\right) \in S \cup(S \times Q), \quad 1 \leqslant i \leqslant m,
\end{gathered}
$$

so that for at most one $1 \leqslant i \leqslant m$,

$$
\chi^{\mathbf{S}}\left(a_{i}\right) \in S \times Q .
$$

Definition 4.12. A non-configuration segment in $A^{\mathrm{S}}$ is a sequence $a_{0}, a_{1}, \ldots$, $a_{k} \in A^{\mathrm{S}}$ such that

$$
a_{0} \rightarrow a_{1} \rightarrow \cdots \rightarrow a_{k}
$$

and either

(a) $\chi^{\mathbf{S}}\left(a_{i}\right) \in S \cup(S \times Q)$, for $0 \leqslant i \leqslant k$, or

(b) $\chi^{\mathbf{S}}\left(a_{0}\right)=\diamond, \chi^{\mathbf{S}}\left(a_{i}\right) \in S \cup(S \times Q), 1 \leqslant i \leqslant k$, or

(c) $\chi^{\mathbf{S}}\left(a_{k}\right)=\varnothing, \chi^{\mathbf{S}}\left(a_{i}\right) \in S \cup(S \times Q), 0 \leqslant i \leqslant k-1$.

Note that the axioms in $\Phi$ imply that, in every line, at most one element has a label in $(S \times Q)$. 
Lemma 4.13. Non-configuration segments generate finite substructures of $\mathbf{S}$ whose $A$-parts, omitting $\infty^{\mathbf{S}}$, have one of the following three forms:

(1)

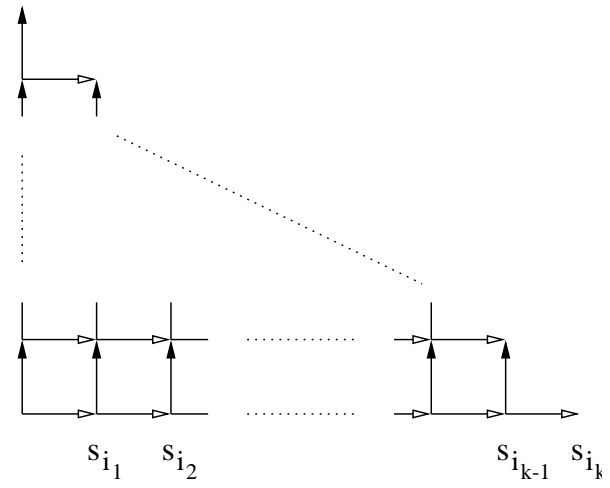

or

(2)

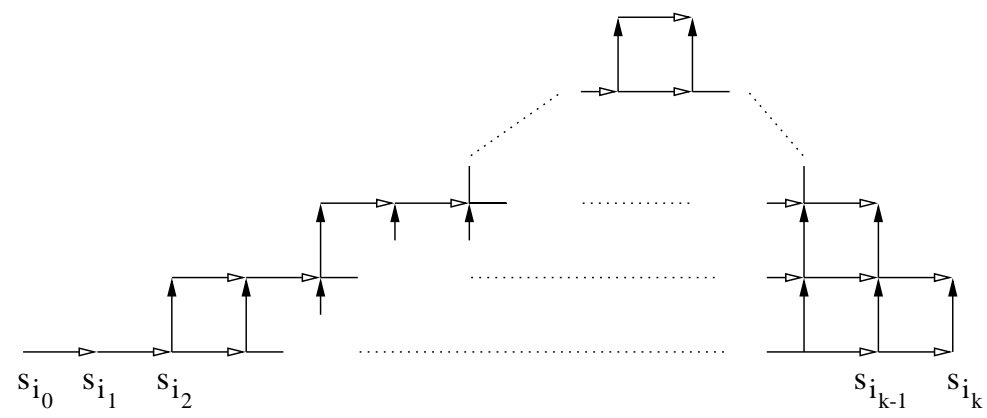

or

(3)

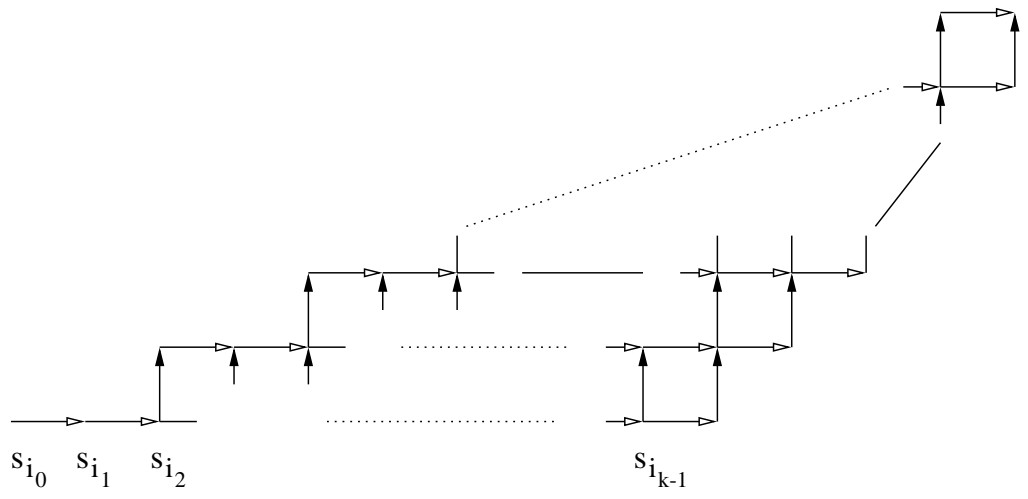


Proof. The proof follows from the axioms which determine the behaviour of diam, $f, g, h$ and bord.

We define the binary relation $\sim$ on $A^{\mathrm{S}}$ to be the equivalence relation on $A^{\mathrm{S}}$ generated by $\rightarrow^{\mathbf{S}} \cup \rightarrow^{\mathbf{S}}$; i.e. $\sim$ is the smallest equivalence relation on $A^{\mathbf{S}}$ containing $\rightarrow \mathbf{S} \cup \rightarrow^{\mathrm{S}}$.

Definition 4.14. A $(\rightarrow \cup \rightarrow)$-connected component (or, simply, $(\rightarrow \cup \rightarrow)-$ component) in $A^{\mathrm{S}}$ is an equivalence class of the binary relation $\sim$.

Let $B$ be an arbitrary $(\rightarrow \cup \rightarrow)$-component in $A^{\mathrm{S}}$, and let

$$
X_{B}=X \cap B .
$$

Let $l_{\mu_{1}}, \ldots, l_{\mu_{k}}$, where $\mu_{1}<\mu_{2}<\cdots<\mu_{k}$, be the lines in $A^{\mathrm{S}}$ in which the elements of $X_{B}$ occur. Clearly, since

$$
\left|X_{B}\right| \leqslant n
$$

where $n$ is the number of elements in $X$, we have

$$
k \leqslant n .
$$

Also, define

$$
X_{i}=X_{B} \cap L_{\mu_{i}}, \quad 1 \leqslant i \leqslant k .
$$

Then,

$$
X_{B}=X_{1} \cup \cdots \cup X_{k} \text {. }
$$

Suppose that

$$
\left|X_{i}\right|=m_{i}, \quad 1 \leqslant i \leqslant k,
$$

which yields

$$
n \geqslant\left|X_{B}\right|=m_{1}+\cdots+m_{k} .
$$

The proof of Lemma 4.6, in fact, shows that if $B$ is a $(\rightarrow \cup \rightarrow)$-component of $A^{\mathrm{S}}$, its projection onto $P_{2}$ is either finite or isomorphic to $\langle\omega, \leqslant\rangle$. This fact will be used in the proof of the following lemma:

Lemma 4.15. Let $B$ be an arbitrary $(\rightarrow \cup \rightarrow)$-component. Let $\mu_{1}, \ldots, \mu_{k}, m_{1}$, $\ldots, m_{k}$ be as defined above.

For all $j<\lambda_{2}$ such that $j \geqslant \mu_{1}$, if none of

$$
B \cap l_{\mu_{1}}, B \cap l_{\mu_{2}}, \ldots, B \cap l_{\mu_{k}}, B \cap l_{j}
$$

are configuration lines, then

$$
\left|B \cap l_{j}\right| \leqslant \max \left(0, \sum_{\mu_{i} \leqslant j} m_{i}-\left(j-\mu_{1}\right)\right) .
$$

Proof. By induction on $j \geqslant \mu_{1}$.

$1^{\circ}$ If $j=\mu_{1}$, the inequality is obvious, for

$$
\left|B \cap l_{j}\right|=\left|B \cap l_{\mu_{1}}\right|=\left|X_{B} \cap l_{\mu_{1}}\right|=m_{1} .
$$


$2^{\circ}$ Suppose $j=i+1$, for some $\mu_{1} \leqslant \lambda_{2}$. Then, $B \cap l_{i}$ will be a non-configuration line; otherwise, $B \cap l_{i}$ will generate a computation structure which will include $B \cap l_{j}$. In this case,

$$
\begin{aligned}
\left|B \cap l_{j}\right| & \leqslant\left|B \cap l_{i}\right|-1 \\
& \leqslant \sum_{\mu_{i} \leqslant k} m_{i}-\left(i-\mu_{1}\right)-1 \\
& \leqslant \sum_{\mu_{i} \leqslant j} m_{i}-\left(j-\mu_{1}\right) .
\end{aligned}
$$

$3^{\circ}$ If $j$ is a limit ordinal $<\lambda_{2}$, then either $j=\mu_{1}$ or $B \cap l_{j}$ is empty. If $j=\mu_{1}$,

$$
\left|B \cap l_{j}\right|=m_{1} .
$$

Thus,

$$
\left|B \cap l_{j}\right| \leqslant \sum_{\mu_{i} \leqslant j} m_{i}-\left(j-\mu_{1}\right)
$$

Corollary 4.16. 1. If $a(\rightarrow \cup \rightarrow)$-component $B$ contains no configuration lines, then $B$ is finite, and

$$
\text { height }(B) \leqslant n, \quad \text { width }(B) \leqslant n^{2} .
$$

2. If $B$ does contain a configuration line, and $j$ is the line number of the first configuration line occurring in $B$, then

$$
j \leqslant \mu_{1}+n \text {. }
$$

Furthermore, the lines in $B$ which precede $B \cap l_{j}$ are bounded in height and width by $n$ and $n^{2}$, respectively.

Proof. Lemma 4.15 tells us that, in the case when $B$ contains no configuration lines, if $j \geqslant \mu_{k}$, then

$$
\left|B \cap l_{j}\right| \leqslant n-\left(j-\mu_{1}\right) .
$$

In case $B$ has an element on $l_{j}$, we see that $j-\mu_{1}<n$. Thus only lines with indices from $\mu_{1}$ to $\mu_{1}+n-1$ can cross $B$. The same applies to all the configuration lines that touch $B$, even if $B$ contains configuration lines.

Hence,

$$
\text { height }(B) \leqslant n,
$$

and

$$
\operatorname{width}(B) \leqslant h e i g h t(B) \cdot \max _{j}\left|B \cap l_{j}\right| \leqslant h e i g h t(B) \cdot n=n^{2} .
$$

Definition 4.17. An infinite $(\rightarrow \cup \rightarrow)$-component will be called standard, if its first line (with respect to the ordering $\leqslant_{2}^{\mathbf{S}}$ ) is a configuration line. Otherwise, it will be called a nonstandard component.

An immediate corollary of the definition above and Lemma 4.15 is the following proposition.

Proposition 4.18. Every infinite $(\rightarrow \cup \rightarrow)$-component is either standard or is a copy of a standard component preceded by finitely many lines. 
Lemma 4.19. If $\mathbf{S}$ is a computation structure, $P_{1}^{\mathbf{S}}$ and $P_{2}^{\mathbf{S}}$ are of order-type $\omega$.

Proof. Let the configuration line generating $A^{\mathrm{S}}$ be

$$
\begin{aligned}
a_{0} & \rightarrow a_{1} \rightarrow \cdots \rightarrow a_{k}, \quad k \geqslant 2, \\
\chi & \mathbf{S}\left(a_{0}\right)=\diamond, \chi^{\mathbf{S}}\left(a_{k}\right)=\varnothing, \chi^{\mathbf{S}}\left(a_{i}\right) \in S \cup(S \times Q), \quad 1 \leqslant i \leqslant k-1 .
\end{aligned}
$$

It is easy to see that, for every $a \in A^{\mathrm{S}}$,

$$
a_{0} \preccurlyeq a .
$$

Hence, in $P_{1}^{\mathbf{S}}$, for every $a \in A^{\mathbf{S}}$, there exist $c_{1}, \ldots, c_{m} \in A^{\mathbf{S}}$, such that

$$
\pi_{1}^{\mathbf{S}}\left(a_{0}\right) \prec_{1} \pi_{1}^{\mathbf{S}}\left(c_{1}\right) \prec_{1} \cdots \prec_{1} \pi_{1}^{\mathbf{S}}\left(c_{m}\right) \prec_{1} \pi_{1}^{\mathbf{S}}(a),
$$

and

$$
P_{1}^{\mathbf{S}}=\pi_{1}^{\mathbf{S}}\left(A^{\mathbf{S}}\right) .
$$

Since the elements $a \in A^{\mathrm{S}}$, for which $\chi^{\mathbf{S}}(a)=\varnothing$, all have distinct $P_{1}$-coordinates, we see that $P_{1}^{\mathbf{S}}$ is countably infinite, and order-isomorphic to $\omega$.

The proof for $\left(P_{2}^{\mathbf{S}}, \leqslant_{2}^{\mathbf{S}}\right)$ is analogous.

In a similar fashion, one can show that, if $\mathbf{S}$ is a structure whose single $(\rightarrow \cup \rightarrow)$ component is infinite nonstandard, and $P_{1}^{\mathbf{S}}, P_{2}^{\mathbf{S}}$ are $\emptyset$-generated, then $P_{1}^{\mathbf{S}}$ and $P_{2}^{\mathbf{S}}$ are of order-type $\omega$.

The results obtained so far can now be summarized in the form of the following proposition.

Proposition 4.20. If $\mathbf{S}=\left(A^{\mathbf{S}}, P_{1}^{\mathbf{S}}, P_{2}^{\mathbf{S}}, C^{\mathbf{S}}\right)$ is a finitely generated structure in $\mathcal{K}$, then

$$
\begin{aligned}
& P_{1}^{\mathbf{S}} \cong a \omega+m_{1}, \quad a \in\{0,1\}, m_{1}<\omega, \\
& P_{2}^{\mathbf{S}} \cong k \omega+m_{2}, \quad k, m_{2}<\omega .
\end{aligned}
$$

Therefore, we have the following theorem:

Theorem 4.21. Every finitely generated structure $\mathbf{S}=\left(A^{\mathbf{S}}, P_{1}^{\mathbf{S}}, P_{2}^{\mathbf{S}}, C^{\mathbf{S}}\right)$ in $\mathcal{K}$ satisfies the following:

(a) $A^{\mathbf{S}}$ is a disjoint union of finitely many $(\rightarrow \cup \rightarrow)$-components, such that each $(\rightarrow \cup \rightarrow)$-component is either

1. finite, or

2. standard,or

3. a component which contains a copy of a standard component preceded by finitely many lines, or

4. $\left\{\infty^{\mathbf{S}}\right\}$.

(b) $P_{1}^{\mathbf{S}}, P_{2}^{\mathbf{S}}$ are well-orderings of types

$$
\begin{array}{lll}
P_{1}^{\mathbf{S}}: & a \omega+m_{1}, & a=0,1, \text { and } m_{1}<\omega, \\
P_{2}^{\mathbf{S}}: & k \omega+m_{2}, & k, m_{2}<\omega .
\end{array}
$$

(c) $C^{\mathbf{S}}$ is finite, and either

1. $c^{\mathbf{S}}=d^{\mathbf{S}}$, or

2. $c^{\mathbf{S}} \neq d^{\mathbf{S}}$.

All the other symbols in $C$ are interpreted by different elements in $C^{\mathbf{S}}$, and, conversely, every element in $C^{\mathbf{S}}$ is the interpretation of some constant symbol in $C$. 
Hence, a typical finitely generated structure in $\mathcal{K}$ can be visualized as:

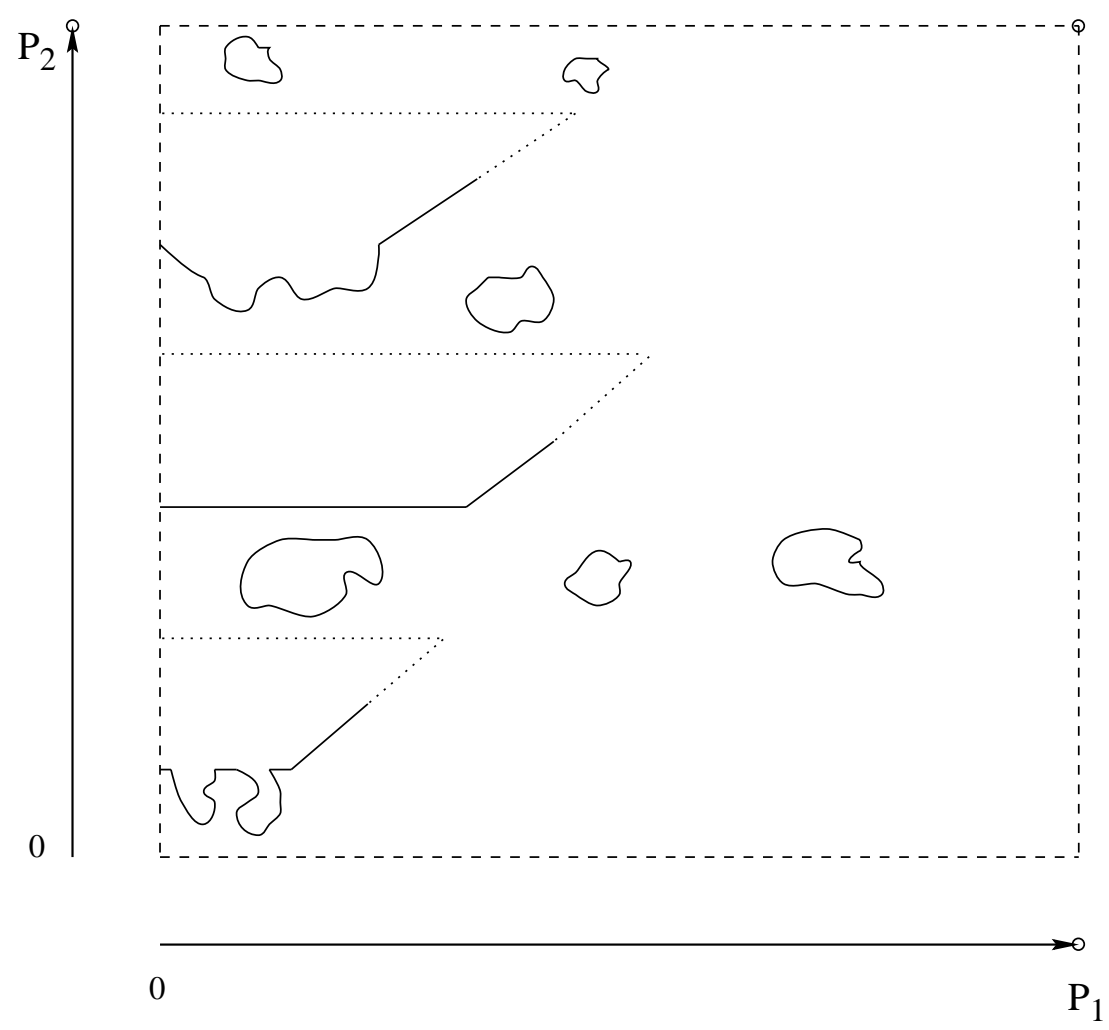

Our next task is to show that $T h_{\forall, n}(\mathcal{K})$ is decidable, for every $n<\omega$. To do that, we construct an algorithm, which, given a universal sentence (or, equivalently, a quantifier-free formula) in $\mathcal{L}$ with at most $n$ variables of each sort, lists all the pairs $(\mathbf{S}, \bar{a})$ of structures in $\mathcal{K}$, generated by at most $n$ elements of each sort and $4 n$-tuples $\bar{a}$ of elements in $\mathbf{S}$ (with $n$ elements of each sort), and checks whether $\bar{a}$ satisfies the given universal sentence in $\mathbf{S}$ or not.

We have already seen that all finite $(\rightarrow \cup \rightarrow)$-components in a structure in $\mathcal{K}$, generated by at most $(n, n, n, 0)$-elements, are bounded in depth and width by $n$ and $n^{2}$, respectively. Therefore, we may assume that every finite $(\rightarrow \cup \rightarrow)$-component is represented by an $n \times n^{2}$-matrix, whose entries are either $\chi$-labels of elements, or some distinguished symbol, say $\mathbf{p}$, if such an element is not present in the finite component. Also, such a matrix contains all the data concerning the relations $\rightarrow$ and $\rightarrow$, restricted to the elements in that finite component. For practical reasons, we also allow for the possibility of the empty finite component.

We may also assume that all the configuration lines (possibly degenerate) of length $\leqslant n$ are given in the form of an $n$-aray whose entries are either $\chi$-labels or

Obviously, a structure in $\mathcal{K}$ generated by at most $n$ elements of each sort can have at most $n(\rightarrow \cup \rightarrow)$-components, not counting $\left\{\infty^{\mathbf{S}}\right\}$. 
Now, if $B_{1}$, and $B_{2}$ are two infinite $(\rightarrow \cup \rightarrow)$-components, such that, for all $x \in B_{1}$, and $y \in B_{2}$,

$$
\begin{gathered}
k \omega+l \leqslant \pi_{2}^{\mathbf{S}}(x)<(k+1) \omega, \\
(k+1) \omega+m \leqslant \pi_{2}^{\mathbf{S}}(y)<(k+2) \omega,
\end{gathered}
$$

where $k, l, m<\omega$, it is easy to see that

$$
0 \leqslant m<n^{2}+n
$$

for there are at most $n$ finite $(\rightarrow \cup \rightarrow)$-components lying "between" $B_{1}$ and $B_{2}$, each of the height at most $n$, plus at most $n$ generators of $P_{2}^{\mathbf{S}}$. For our purposes, the bound on $m$ can be given by $2 n^{2}$, which will make it easier to work with.

Our strategy will be to construct first, for every $0 \leqslant k \leqslant n$, a procedure which lists all structures in $\mathcal{K}$ (up to isomorphism) with precisely $k$ infinite $(\rightarrow \cup \rightarrow)$ components, and then use these procedures to list all structures in $\mathcal{K}$ (up to isomorphism) with at most $n(\rightarrow \cup \rightarrow)$-components, excluding $\left\{\infty^{\mathbf{S}}\right\}$.

If $k=0$, then $P_{1}^{\mathbf{S}}$, and $P_{2}^{\mathbf{S}}$ are of order-types $m_{1}$, and $m_{2}$, respectively and

$$
\begin{aligned}
& m_{1} \leqslant n \cdot n^{2}+n \leqslant 2 n^{3}, \\
& m_{2} \leqslant n \cdot n+n \leqslant 2 n^{2},
\end{aligned}
$$

since there are at most $n$ components, all of which are finite, and the procedure for $k=0$ can be realized through a brute force search through all the possibilities for at most $n$ finite $(\rightarrow \cup \rightarrow)$-components.

If $k \neq 0$, then $P_{1}^{\mathbf{S}}$ and $P_{2}^{\mathbf{S}}$ have order-types

$$
\begin{aligned}
\omega+m_{1}, & m_{1} \leqslant n \cdot n^{2}+n \leqslant 2 n^{3}, \\
k \omega+m_{2}, & m_{2} \leqslant n \cdot n+n \leqslant 2 n^{2} .
\end{aligned}
$$

Thus, given $k$, the elements of $A^{\mathbf{S}}$ have horizontal and vertical coordinates within the following ranges:

$$
\begin{array}{ll}
P_{1}: & 0 \leqslant x \leqslant \omega+2 n^{3}, \\
P_{2}: & 0 \leqslant x \leqslant k \omega+2 n^{2} .
\end{array}
$$

We proceed to the informal description of the algorithm, when $k \neq 0$.

$1^{\circ}$ Let $C_{1}, \ldots, C_{l}$ be all sequences of $k$ configuration lines of length $<n$.

Let $\mathcal{P}_{i}$ be the procedure which, given

$$
C_{i}=\left\langle l_{i_{1}}, \ldots, l_{i_{k}}\right\rangle
$$

lists all $\mathcal{K}$-structures for which the standard parts of $k$ infinite $(\rightarrow \cup \rightarrow)$-components start with $l_{i_{1}}, \ldots, l_{i_{k}}$. Then, once all $\mathcal{P}_{i}, 1 \leqslant i \leqslant l$, have been constructed, we can list all structures in $\mathcal{K}$ with precisely $k$ infinite components, in the following way: list the first structure produced by $\mathcal{P}_{1}$, next, the first structure listed by $\mathcal{P}_{2}$, etc. Finally, after the first structure produced by $\mathcal{P}_{l}$ has been listed, proceed to the second structure listed by $\mathcal{P}_{1}$, the second structure listed by $\mathcal{P}_{2}$, and so on.

Thus, our task reduces to constructing $\mathcal{P}_{i}$ for $1 \leqslant i \leqslant l$.

$2^{\circ}$ Let us focus our attention on a particular $C_{i}(1 \leqslant i \leqslant l)$. Suppose that

$$
C_{i}=\left\langle l_{i_{1}}, \ldots, l_{i_{k}}\right\rangle .
$$




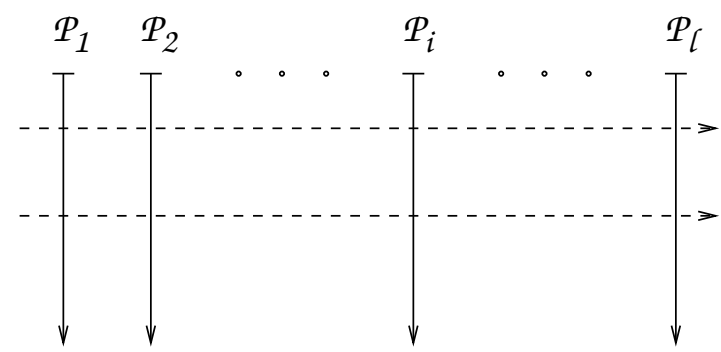

Then, we assign $P_{2}$-coordinates to these $k$ lines in the following manner:

$$
\begin{aligned}
l_{i_{1}} & \mapsto 2 n^{2} \\
l_{i_{2}} & \mapsto \omega+2 n^{2} \\
\quad & \\
l_{i_{k}} & \mapsto(k-1) \omega+2 n^{2} .
\end{aligned}
$$

Since in an $(n, n, n, 0)$-generated structure there are only finitely many possible finite parts in nonstandard infinite components (including the empty finite part) $N_{1}, \ldots, N_{m}$, there are only finitely many $k$-sequences of them, say $D_{1}, \ldots, D_{p}$.

Now, for the specified $C_{i}$, let

$$
D_{j}=\left\langle N_{j_{1}}, \ldots, N_{j_{k}}\right\rangle .
$$

We "position" each $N_{j_{i}}$ in the coordinate lattice by selecting the $P_{2}$-coordinate $y_{j_{i}}$ for the bottom line of each of them, so that

$$
(i-1) \omega \leqslant y_{j_{i}} \leqslant(i-1) \omega+2 n^{2}-1 .
$$

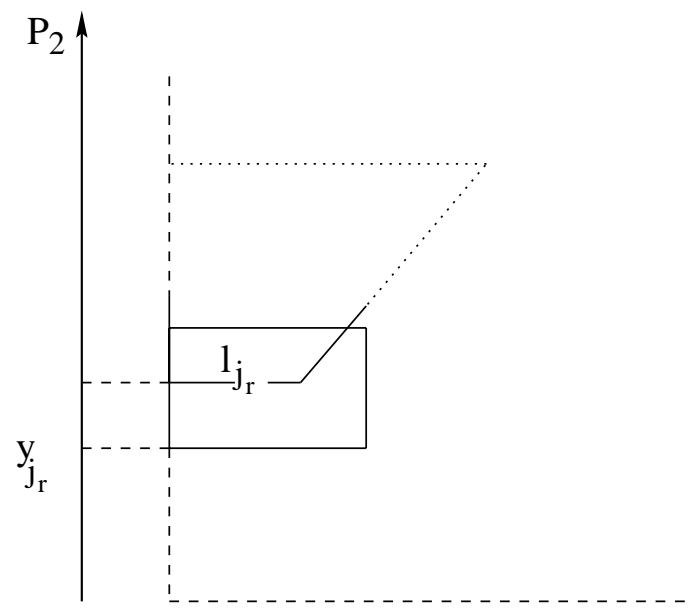

The next step is to check for the compatibility of each $N_{j_{r}}$ with the corresponding standard component which starts with $l_{j_{r}}$. 
- If $N_{j_{r}}=\emptyset$, the output is: " $N_{j_{r}}$ is compatible with $l_{j_{r}}$ ".

- Otherwise, if $N_{j_{r}} \neq \emptyset$, using the coordinatization of $A^{\mathrm{S}}$, we can effectively determine whether $N_{j_{r}}$ and the standard part produced by $l_{j_{r}}$ overlap in an allowable way.

Next, we need to specify the finite $(\rightarrow \cup \rightarrow)$-components in the structure (at most $n-k$ of them; but, since we allow for the empty component, we may assume that there are precisely $n-k$ of them), and their position.

Again, there are only finitely many types of such finite components (apart from their location). Let $E_{1}, \ldots, E_{s}$ be all $(n-k)$-sequences of finite components in a structure generated by at most $(n, n, n, 0)$-elements.

Now, given $C_{i}$ and $D_{j}$, consider some $E_{l}(1 \leqslant l \leqslant s)$, where

$$
E_{l}=\left\langle B_{l_{1}}, \ldots, B_{l_{n-k}}\right\rangle .
$$

We shall specify the positions of $B_{l_{r}}(1 \leqslant r \leqslant n-k)$, by selecting the pair $\langle x, y\rangle$ of coordinates for the left bottom entry of the matrix representing $B_{l_{r}}$.

Also,

$$
\begin{gathered}
1 \leqslant x \leqslant \omega+n^{3}, \\
y \in\left[0,2 n^{2}-1\right] \cup\left[\omega, \omega+2 n^{2}-1\right] \cup \cdots \cup\left[k \omega, k \omega+2 n^{2}\right] .
\end{gathered}
$$

Obviously, all such $(n-k)$-tuples of pairs $\langle x, y\rangle$ can be effectively enumerated, and let $\mathcal{Q}$ be the procedure which performs that task.

After the procedure $\mathcal{Q}$ outputs a $(n-k)$-tuple

$$
\left\langle\left\langle x_{1}, y_{1}\right\rangle, \ldots,\left\langle x_{n-k}, y_{n-k}\right\rangle\right\rangle,
$$

assign $\left\langle x_{r}, y_{r}\right\rangle(1 \leqslant r \leqslant n-k)$ to $B_{l_{r}}$ as the coordinates of its left bottom element, and, for each pair $l_{i_{q}}$ and $N_{j_{m}}(1 \leqslant q, m \leqslant k)$, check whether $B_{l_{r}}(1 \leqslant r \leqslant n-k)$ overlaps with any of $l_{i_{q}}, N_{j_{m}}$. If the answer is negative, for every $B_{l_{r}}(1 \leqslant r \leqslant n-k)$, and $E_{l}$ is an allowable disposition of finite components in the structure (together with the locations of $E_{l}$, given by $\mathcal{Q}$ ), in which all infinite components are specified by $C_{i}$ and $D_{j}$.

Hence, the procedure $\mathcal{P}_{i}$ which, given $k(0 \leqslant k \leqslant n)$ and $C_{i}=\left\langle l_{i_{1}}, \ldots, l_{i_{k}}\right\rangle$ $(1 \leqslant i \leqslant l)$ lists all structures, up to isomorphism, in which the standard parts of infinite configurations start with $l_{i_{1}}, \ldots, l_{i_{k}}$, respectively, is given by the following:

(1) Choose $1 \leqslant j \leqslant p$; i.e. $D_{j}=\left\langle N_{j_{1}}, \ldots, N_{j_{k}}\right\rangle$.

(2) For all possible choices of $k$-tuples $\bar{y}=\left\langle y_{j_{1}}, \ldots, y_{j_{k}}\right\rangle$, such that

$$
(i-1) \omega \leqslant y_{j_{i}} \leqslant(i-1) \omega+3 n^{2}-1,
$$

assign $y_{j_{r}}$ to $N_{j_{r}}$ as the $P_{2}$-coordinate of its bottom line and check whether each $N_{j_{r}}$ is compatible with $l_{j_{r}}(1 \leqslant r \leqslant k)$.

(3) For each $D_{j}$ and $\bar{y}$, for which $D_{j}$ and $\bar{y}$ are compatible with $C_{i}$, list all the structures specified by $C_{i}, D_{j}$, and $\bar{y}$ in the following way:

- choose $E_{l}(1 \leqslant l \leqslant s)$,

- use $\mathcal{Q}$ to generate all the allowable structures with $E_{l}$ as finite components.

(4) The last fact that we need in order to specify the structure completely is whether $c=d$ in the structure or not. If $T$, started on any of $l_{i_{1}}, \ldots, l_{i_{k}}$, halts, we are forced to have $c^{\mathbf{S}}=d^{\mathbf{S}}$; otherwise, there are two structures with $C_{i}, D_{j}, E_{l}$, and $\bar{y}, s$ as described above, one for which $c^{\mathbf{S}}=d^{\mathbf{S}}$, and the other for which $c^{\mathbf{S}} \neq d^{\mathbf{S}}$. 


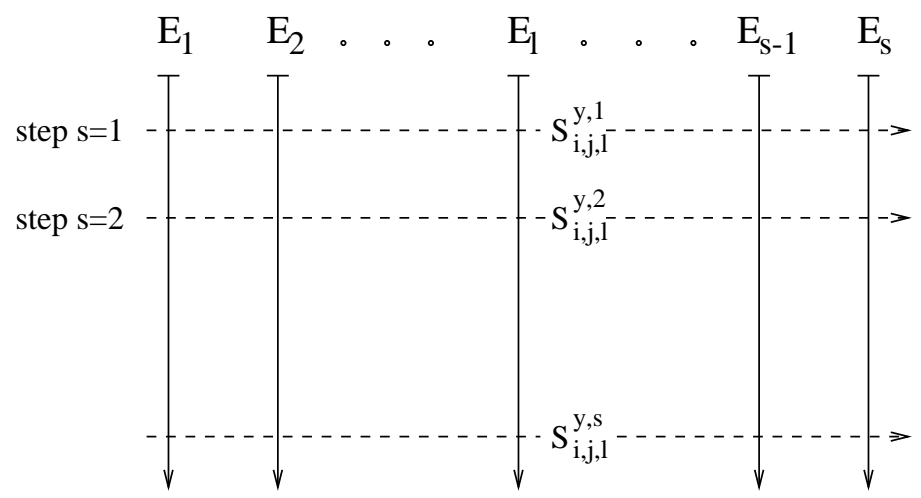

Thus, after generating $S_{i, j, l}^{r, s}$ (here, $r$ is the corresponding code for $\bar{y}$ ) in step (3), we check whether $T$ started on any of $l_{i_{1}}, \ldots, l_{i_{k}}$ halts. This is possible, since

$$
\left\{q: T \text { started on } l_{i_{q}} \text { halts, } 1 \leqslant q \leqslant k\right\}
$$

is finite, and hence, recursive.

If the answer is positive, we list $S_{i, j, l}^{r, s,=}$; otherwise, we list both $S_{i, j, l}^{r, s,=}$ and $S_{i, j, l}^{r, s, \neq}$. Finally, we include $k$ (the number of infinite components in the structure) in the code, which now has the form

$$
S_{k, i, j, l}^{r, s,=}
$$

or

$$
S_{k, i, j, l}^{r, s, \neq} \text {. }
$$

This gives an enumeration, which includes all $(n, n, n, 0)$-generated structures in $\mathcal{K}$, which is effective in the following sense: given the code $S_{k, i, j, l}^{r, s, *}$, where $* \in\{=, \neq\}$, we can obtain the complete specification of the structure it represents.

Now, there is an effective procedure listing all $4 n$-tuples

$$
\left\langle a_{1}, \ldots, a_{n}, b_{1}, \ldots, b_{n}, c_{1}, \ldots, c_{n}, d_{1}, \ldots, d_{n}\right\rangle,
$$

such that $a_{i}=\left\langle x_{i}, y_{i}\right\rangle$, where

$$
\begin{aligned}
& 0 \leqslant x_{i} \leqslant \omega+3 n^{3}, \\
& 0 \leqslant y_{i} \leqslant n \omega+3 n^{2} .
\end{aligned}
$$

(These $n$ elements represent the elements of $A^{\mathrm{S}}$ via their coordinates.) Next $n$ entries $b_{1}, \ldots, b_{n}$ are elements of $\omega+3 n^{3}$ (elements of $\left.P_{1}^{\mathbf{S}}\right), c_{1}, \ldots, c_{n}$ are in $n \omega+2 n^{3}$ (elements of $P_{2}^{\mathbf{S}}$ ), while $d_{1}, \ldots, d_{n} \in C^{\mathbf{S}}$.

If $\mathbf{S}_{1}, \mathbf{S}_{2}, \mathbf{S}_{3}, \ldots$ is the listing of the structures in $\mathcal{K}$ having at most $n$ components (excluding $\left\{\infty^{\mathbf{S}}\right\}$ ), as described in the preceding paragraphs, and $\sigma_{1}, \sigma_{2}, \ldots$ is the listing of all the $4 n$-tuples as above, we check whether $\sigma_{i}$ is in $\mathbf{S}_{j}$

using the information contained in the code for $\mathbf{S}_{j}$ and the coordinatization of $A^{\mathbf{S}_{j}}$. This produces an enumeration of descriptions of pairs $\langle\mathbf{S}, \bar{a}\rangle$, where

1. for every such $\langle\mathbf{S}, \bar{a}\rangle, \mathbf{S} \in \mathcal{K}$ and $\bar{a}$ is an $(n, n, n, 0)$-tuple from $\mathbf{S}$;

2. conversely, if $\mathbf{S} \in \mathcal{K}, \bar{a}$ is an $(n, n, n, 0)$-tuple from $\mathbf{S}$, and $\mathbf{S}$ is generated by $\bar{a}$, then the enumeration contains a description of $\langle\mathbf{S}, \bar{a}\rangle$. 


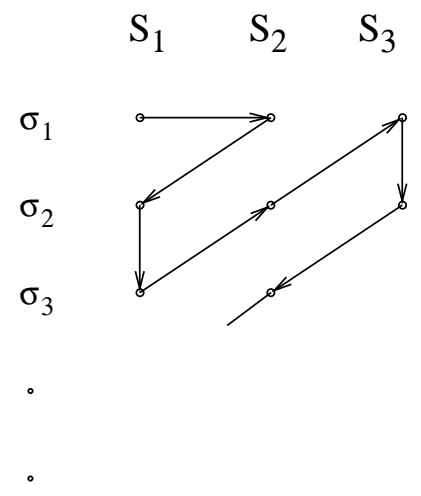

The code for $\mathbf{S}$ provides us with the specification of $\mathbf{S}$, with one possible difficulty: the code for $\mathbf{S}$ specifies only the initial line of the standard part of an infinite $(\rightarrow \cup \rightarrow)$-component in $A^{\mathrm{S}}$. In order to check the nonvalidity of a given quantifierfree formula $\phi$ in $\mathbf{S}$, when the variables of $\phi$ are substituted with the corresponding entries from $\sigma$, we must be able to recover a "sufficiently large" standard part of any infinite $(\rightarrow \cup \rightarrow)$-component.

Let $B$ be a standard component, whose initial line is given by

$$
\begin{gathered}
a_{0} \rightarrow a_{1} \rightarrow \cdots \rightarrow a_{k} \rightarrow a_{k+1}, \\
\chi^{\mathbf{S}}\left(a_{0}\right)=\diamond, \chi^{\mathbf{S}}\left(a_{1}\right) \in S \cup(S \times Q), 1 \leqslant i \leqslant k, \\
\chi^{\mathbf{S}}\left(a_{k+1}\right)=\varnothing, \\
\pi_{1}^{\mathbf{S}}\left(a_{0}\right), \ldots, \pi_{1}^{\mathbf{S}}\left(a_{k+1}\right)=k+1, \\
\pi_{2}^{\mathbf{S}}\left(a_{0}\right)=\cdots=\pi_{2}^{\mathbf{S}}\left(a_{k+1}\right)=m, \text { for some } m<\lambda_{2} .
\end{gathered}
$$

Set

$$
B^{(0)}=\left\langle A^{(0)}, \rightarrow^{(0)}, \rightarrow^{(0)}\right\rangle,
$$

where

$$
\begin{aligned}
& A^{(0)}=\left\{\left\langle a_{i}, \chi^{\mathbf{S}}\left(a_{i}\right), \pi_{1}^{\mathbf{S}}\left(a_{i}\right), \pi_{2}^{\mathbf{S}}\left(a_{i}\right)\right\rangle: 0 \leqslant i \leqslant k+1\right\}, \\
& \rightarrow^{(0)}=\left\{\left\langle a_{j}, a_{j+1}\right\rangle: 0 \leqslant j \leqslant k\right\}, \\
& \rightarrow^{(0)}=\emptyset .
\end{aligned}
$$

Given $B^{(n)}=\left\langle A^{(n)}, \rightarrow^{(n)}, \rightarrow^{(n)}\right\rangle$, we define $B^{(n+1)}$ in the following way:

$$
B^{(n+1)}=\left\langle A^{(n+1)}, \rightarrow^{(n+1)}, \rightarrow^{(n+1)}\right\rangle,
$$

where

$$
\begin{gathered}
A^{(n+1)}=A^{(n)} \cup\left\{\left\langleF^{\mathbf{S}}\left(b_{1}, \ldots, b_{m}\right), \chi^{\mathbf{S}}\left(F^{\mathbf{S}}\left(b_{1}, \ldots, b_{m}\right)\right), \pi_{1}^{\mathbf{S}}\left(b_{F}\right)\left(\text { or } \pi_{1}^{\mathbf{S}}\left(b_{F}\right)+1\right.\right.\right. \\
\text { when } \left.\left.F=\text { bord }), \pi_{2}^{\mathbf{S}}\left(b_{F}\right)+1\right\rangle \mid F: A^{m} \rightarrow A, b_{1}, \ldots, b_{m} \in A^{(n)}\right\}
\end{gathered}
$$

where

$$
b_{F}= \begin{cases}b_{1}, & F=d i a m \\ b_{2}, & F=g, h \text { or bord } \\ b_{3}, & F=f\end{cases}
$$


and

$$
\begin{aligned}
& \rightarrow^{(n+1)}=\rightarrow^{(n)} \cup\left\{\langle a, b\rangle \in\left(A^{(n+1)} \backslash A^{(n)}\right)^{2}: \pi_{1}^{\mathbf{S}}(b)=\pi_{1}^{\mathbf{S}}(a)+1\right\}, \\
& \rightarrow^{(n+1)}=\rightarrow^{(n)} \cup\left\{\langle a, b\rangle \in A^{(n)} \times A^{(n+1)}: \pi_{1}^{\mathbf{S}}(a)=\pi_{1}^{\mathbf{S}}(b)\right\} .
\end{aligned}
$$

Then,

$$
B=\bigcup_{n<\omega} B^{(n)},
$$

and a "sufficiently large" part of $B$ can be effectively recovered by constructing $B^{(n)}, n<\omega$

Therefore, the algorithm that, given a quantifier-free formula in $\mathcal{L}$ with at most $n$ variables of each sort, checks whether the formula belongs to $T h_{\forall, n}(\mathcal{K})$ or not, is given by the following: on one hand, list all the theorems which can be deduced from $T$; on the other hand, list the pairs $(\mathbf{S}, \sigma)$, as described above, and check whether the formula is false in $\mathbf{S}$ when its variables are substituted by the entries of $\sigma$. If so, list it. Eventually, the formula will appear in one of these two lists.

Hence, we have the following theorem:

Theorem 4.22. $T h_{\forall, n}(\mathcal{K})$ is decidable, for every $n<\omega$.

This finishes the proof that the class $\mathcal{K}$ is universally pseudorecursive.

\section{Appendix: The list of Axioms for $\mathcal{K}$}

1. Axioms which assert that $\leqslant_{i}$ is a linear ordering with the maximal element $\infty_{i}$ :

- $\left(\forall x \in P_{i}\right)\left(x \leq_{i} x\right)$,

- $\left(\forall x \in P_{i}\right)\left(\forall y \in P_{i}\right)\left(x \leq_{i} y \wedge y \leq_{i} x \Rightarrow x=y\right)$,

- $\left(\forall x \in P_{i}\right)\left(\forall y \in P_{i}\right)\left(\forall z \in P_{i}\right)\left(x \leq_{i} y \wedge y \leq_{i} z \Rightarrow x \leq_{i} z\right.$,

- $\left(\forall x \in P_{i}\right)\left(\forall y \in P_{i}\right)\left(x \leq_{i} y \vee y \leq_{i} x\right)$,

- $\left(\forall x \in P_{i}\right)\left(x \leq_{i} \infty_{i}\right)$.

2. Axioms which describe $\pi_{i}$ and their relation to $\rightarrow$ and $\rightarrow$ :

- $(\forall x \in A)\left(\forall y \in P_{1}\right)\left(\chi(x)=\diamond \Rightarrow \pi_{1}(x) \leq_{1} y\right)$,

- $(\forall x \in A)(\forall y \in A)\left(\chi(x)=\varnothing \wedge \pi_{2}(x)=\pi_{2}(y) \Rightarrow \pi_{1}(y) \leq_{1} \pi_{1}(x)\right)$,

- $(\forall x \in A)(\forall y \in A)\left(\pi_{1}(x)=\pi_{1}(y) \wedge \pi_{2}(x)=\pi_{2}(y) \Rightarrow x=y\right)$,

- $(\forall x \in A)(\forall y \in A)\left(\chi(x)=\diamond \wedge \pi_{1}(x)=\pi_{1}(y) \Rightarrow \chi(y)=\diamond\right)$,

- $(\forall x \in A)\left(\pi_{i}(x)=\infty_{i} \Leftrightarrow x=\infty\right)$,

- $(\forall x \in A)(\forall y \in A)\left(\forall z \in P_{1}\right)\left(x \rightarrow y \Rightarrow \pi_{1}(x)<_{1} \pi_{1}(y) \wedge \neg\left(\pi_{1}(x)<_{1} z<_{1}\right.\right.$ $\left.\left.\pi_{1}(y)\right) \wedge \pi_{2}(x)=\pi_{2}(y)\right)$

- $(\forall x \in A)(\forall y \in A)\left(\forall z \in P_{2}\right)\left(x \rightarrow y \Rightarrow \pi_{2}(x)<_{2} \pi_{2}(y) \wedge \neg\left(\pi_{2}(x)<_{2} z<_{2}\right.\right.$ $\left.\left.\pi_{2}(y)\right) \wedge \pi_{1}(x)=\pi_{1}(y)\right)$

- $(\forall x \in A)(\forall y \in A)(\forall z \in A)(\forall u \in A)(x \rightarrow y \wedge x \rightarrow z \wedge y \rightarrow u \Rightarrow z \rightarrow u)$,

- $(\forall x \in A)(\forall y \in A)(\forall z \in A)(\forall u \in A)(x \rightarrow y \wedge x \rightarrow z \wedge z \rightarrow u \Rightarrow y \rightarrow u)$,

- $(\forall x \in A)(\forall y \in A)(\forall z \in A)(\forall u \in A)(x \rightarrow y \wedge z \rightarrow u \wedge y \rightarrow u \Rightarrow x \rightarrow z)$,

- $(\forall x \in A)(\forall y \in A)(\forall z \in A)(\forall u \in A)(x \rightarrow z \wedge z \rightarrow u \wedge y \rightarrow u \Rightarrow x \rightarrow y)$.

3. Axioms which describe the labelling by $\chi$ and the detection of the halting state:

- For each $s \in S$

$$
(\forall x \in A)\left(\chi(x)=\left(s, q_{0}\right) \Rightarrow c=d\right),
$$

- $(\forall x \in C)\left(\bigvee\left\{x=s: s \in S \cup(S \times Q) \cup\left\{c, d, \infty^{\prime}\right\}\right\} \wedge \wedge\left\{c_{1} \neq c_{2}: c_{1}, c_{2} \in C\right.\right.$, except for $\left.\left\{c_{1}, c_{2}\right\}=\{c, d\}\right\}$ ), 
- $(\forall x \in A)\left(\bigvee\left\{\chi(x)=c: c \in S \cup(S \times Q) \cup\left\{\diamond, \varnothing, \infty^{\prime}\right\}\right\}\right)$,

- $(\forall x \in A)(\forall y \in A)\left(\pi_{2}(x)=\pi_{2}(y) \wedge \chi(x)=\chi(y)=\varnothing \Rightarrow x=y\right)$,

- $(\forall x \in A)\left(\chi(x)=\infty^{\prime} \Leftrightarrow x=\infty\right)$,

- For $(s, q),\left(s^{\prime}, q^{\prime}\right) \in S \times Q$ :

$(\forall x \in A)(\forall y \in A)\left(\pi_{2}(x)=\pi_{2}(y) \wedge \chi(x)=(s, q) \wedge \chi(y)=\left(s^{\prime}, q^{\prime}\right) \Rightarrow x=y\right)$,

- $(\forall x \in A) \neg(x \rightarrow \infty \vee x \rightarrow \infty)$,

- $(\forall x \in A)(\forall y \in A) \neg(x \rightarrow y \wedge \chi(y)=\varnothing)$,

- $(\forall x \in A)(\forall y \in A) \neg(\chi(x)=\diamond \wedge \chi(y)=\varnothing \wedge x \rightarrow y)$,

4. Axioms which describe bord operation:

- $(\forall x \in A)(\forall y \in A)(x \rightarrow y \wedge \chi(y)=\varnothing \Rightarrow g(x, y) \rightarrow \operatorname{bord}(x, y) \wedge$ $\chi(\operatorname{bord}(x, y))=\varnothing)$,

- $(\forall x \in A)(\forall y \in A)(\neg x \rightarrow y \vee \chi(y) \neq \varnothing \Rightarrow g(x, y)=\infty)$.

5. Axioms which describe operation $g$ :

- $(\forall x \in A)(\forall y \in A)(x \rightarrow y \wedge \chi(y)=\varnothing \wedge \chi(x)=s(s \in S) \Rightarrow y \rightarrow g(x, y)$ $\wedge \chi(g(x, y))=B)$

- If $T(s, q)=\left(s^{\prime}, R, q^{\prime}\right)$ :

$(\forall x \in A)(\forall y \in A)(x \rightarrow y \wedge \chi(y)=\varnothing \wedge \chi(x)=(s, q) \Rightarrow y \rightarrow g(x, y) \wedge$ $\left.\chi(g(x, y))=\left(B, q^{\prime}\right)\right)$

- If $T(s, q)=\left(s^{\prime}, L, q^{\prime}\right)$ or $T(s, q)=\left(s^{\prime}, N, q^{\prime}\right)$ :

$(\forall x \in A)(\forall y \in A)(x \rightarrow y \wedge \chi(y)=\varnothing \wedge \chi(x)=(s, q) \Rightarrow y \rightarrow g(x, y) \wedge$ $\chi(g(x, y))=B)$.

6. Axioms which describe diam operation:

- $(\forall x \in A)(\forall y \in A)(x \rightarrow y \wedge \chi(x)=\diamond \Rightarrow x \rightarrow \operatorname{diam}(x, y) \wedge \chi(\operatorname{diam}(x, y))$ $=\diamond)$

- $(\forall x \in A)(\forall y \in A)(\neg x \rightarrow y \vee \chi(x) \neq \diamond \Rightarrow \operatorname{diam}(x, y)=\infty)$.

7. Axioms which describe the operation $f$ :

- For $s_{i} \in S$ :

$(\forall x \in A)(\forall y \in A)(\forall z \in A)(\forall w \in A)\left(x \rightarrow y \rightarrow z \rightarrow w \wedge\left(\left(\chi(x)=s_{1}\right) \vee\right.\right.$ $(\chi(x)=\diamond)) \wedge \chi(y)=s_{2} \wedge \chi(z)=s_{3} \wedge\left(\left(\chi(w)=s_{4}\right) \vee(\chi(w)=\varnothing)\right) \Rightarrow$ $\left.z \rightarrow f(x, y, z, w) \wedge \chi(f(x, y, z, w))=s_{3}\right)$,

- If $T\left(s_{4}, q\right)=\left(s^{\prime}, N, q^{\prime}\right)$, or $T\left(s_{4}, q\right)=\left(s^{\prime}, R, q^{\prime}\right)$ :

$(\forall x \in A)(\forall y \in A)(\forall z \in A)(\forall w \in A)\left(x \rightarrow y \rightarrow z \rightarrow w \wedge\left(\left(\chi(x)=s_{1}\right) \vee\right.\right.$ $(\chi(x)=\diamond)) \wedge \chi(y)=s_{2} \wedge \chi(z)=s_{3} \wedge \chi(w)=\left(s_{4}, q\right) \Rightarrow z \rightarrow f(x, y, z, w)$ $\left.\wedge \chi(f(x, y, z, w))=s_{3}\right)$.

- If $T\left(s_{4}, q\right)=\left(s^{\prime}, L, q^{\prime}\right)$ :

$(\forall x \in A)(\forall y \in A)(\forall z \in A)(\forall w \in A)\left(x \rightarrow y \rightarrow z \rightarrow w \wedge\left(\left(\chi(x)=s_{1}\right) \vee\right.\right.$ $(\chi(x)=\diamond)) \wedge \chi(y)=s_{2} \wedge \chi(z)=s_{3} \wedge \chi(w)=\left(s_{4}, q\right) \Rightarrow z \rightarrow f(x, y, z, w)$ $\left.\wedge \chi(f(x, y, z, w))=\left(s_{3}, q^{\prime}\right)\right)$.

- If $T\left(s_{3}, q\right)=\left(s^{\prime}, N, q^{\prime}\right)$ :

$(\forall x \in A)(\forall y \in A)(\forall z \in A)(\forall w \in A)\left(x \rightarrow y \rightarrow z \rightarrow w \wedge\left(\left(\chi(x)=s_{1}\right) \vee\right.\right.$ $(\chi(x)=\diamond)) \wedge \chi(y)=s_{2} \wedge \chi(z)=\left(s_{3}, q\right) \wedge\left(\left(\chi(w)=s_{4}\right) \vee(\chi(w)=\right.$ ○) $\left.) \Rightarrow z \rightarrow f(x, y, z, w) \wedge \chi(f(x, y, z, w))=\left(s^{\prime}, q^{\prime}\right)\right)$.

- If $T\left(s_{3}, q\right)=\left(s^{\prime}, L, q^{\prime}\right)$ or $T\left(s_{3}, q\right)=\left(s^{\prime}, R, q^{\prime}\right)$ :

$(\forall x \in A)(\forall y \in A)(\forall z \in A)(\forall w \in A)\left(x \rightarrow y \rightarrow z \rightarrow w \wedge\left(\left(\chi(x)=s_{1}\right) \vee\right.\right.$ $(\chi(x)=\diamond)) \wedge \chi(y)=s_{2} \wedge \chi(z)=\left(s_{3}, q\right) \wedge\left(\left(\chi(w)=s_{4}\right) \vee(\chi(w)=\right.$ ○) $\left.\Rightarrow z \rightarrow f(x, y, z, w) \wedge \chi(f(x, y, z, w))=s^{\prime}\right)$.

- If $T\left(s_{2}, q\right)=\left(s^{\prime}, L, q^{\prime}\right)$ or $T\left(s_{2}, q\right)=\left(s^{\prime}, N, q^{\prime}\right)$ : 
$(\forall x \in A)(\forall y \in A)(\forall z \in A)(\forall w \in A)\left(x \rightarrow y \rightarrow z \rightarrow w \wedge\left(\left(\chi(x)=s_{1}\right) \vee\right.\right.$ $(\chi(x)=\diamond)) \wedge \chi(y)=\left(s_{2}, q\right) \wedge \chi(z)=s_{3} \wedge\left(\left(\chi(w)=s_{4}\right) \vee(\chi(w)=\right.$ ○) $\left.\Rightarrow z \rightarrow f(x, y, z, w) \wedge \chi(f(x, y, z, w))=s_{3}\right)$.

- If $T\left(s_{2}, q\right)=\left(s^{\prime}, R, q^{\prime}\right)$ :

$(\forall x \in A)(\forall y \in A)(\forall z \in A)(\forall w \in A)\left(x \rightarrow y \rightarrow z \rightarrow w \wedge\left(\left(\chi(x)=s_{1}\right) \vee\right.\right.$ $(\chi(x)=\diamond)) \wedge \chi(y)=\left(s_{2}, q\right) \wedge \chi(z)=s_{3} \wedge\left(\left(\chi(w)=s_{4}\right) \vee(\chi(w)=\right.$ ๑) $\left.\Rightarrow z \rightarrow f(x, y, z, w) \wedge \chi(f(x, y, z, w))=\left(s_{3}, q^{\prime}\right)\right)$.

- $(\forall x \in A)(\forall y \in A)(\forall z \in A)(\forall w \in A)\left(x \rightarrow y \rightarrow z \rightarrow w \wedge\left(\chi(x)=\left(s_{1}, q\right) \wedge\right.\right.$ $\left.\chi(y)=s_{2} \wedge \chi(z)=s_{3} \wedge\left(\left(\chi(w)=s_{4}\right) \vee(\chi(w)=\varnothing)\right)\right) \Rightarrow z \rightarrow f(x, y, z, w)$ $\left.\wedge \chi(f(x, y, z, w))=s_{3}\right)$,

- $(\forall x \in A)(\forall y \in A)(\forall z \in A)(\forall w \in A)$ (all previous antecedents in this group are false $\Rightarrow f(x, y, z, w)=\infty)$.

8. Axioms which describe operation $h$ :

- $(\forall x \in A)(\forall y \in A)(\forall z \in A)\left(x \rightarrow y \rightarrow z \wedge \chi(x)=\diamond \wedge \chi(y)=s_{2} \wedge\right.$ $\left.\left(\left(\chi(z)=s_{3}\right) \vee(\chi(z)=\varnothing)\right) \Rightarrow y \rightarrow h(x, y, z) \wedge \chi(h(x, y, z))=s_{2}\right)$.

- If $T\left(s_{3}, q\right)=\left(s^{\prime}, N, q^{\prime}\right)$ or $\left(s^{\prime}, R, q^{\prime}\right)$ :

$(\forall x \in A)(\forall y \in A)(\forall z \in A)\left(x \rightarrow y \rightarrow z \wedge \chi(x)=\diamond \wedge \chi(y)=s_{2} \wedge\right.$ $\left.\chi(z)=\left(s_{3}, q\right) \Rightarrow y \rightarrow h(x, y, z) \wedge \chi(h(x, y, z))=s_{2}\right)$.

- If $T\left(s_{3}, q\right)=\left(s^{\prime}, L, q^{\prime}\right)$ :

$(\forall x \in A)(\forall y \in A)(\forall z \in A)\left(x \rightarrow y \rightarrow z \wedge \chi(x)=\diamond \wedge \chi(y)=s_{2} \wedge\right.$ $\left.\chi(z)=\left(s_{3}, q\right) \Rightarrow y \rightarrow h(x, y, z) \wedge \chi(h(x, y, z))=\left(s_{2}, q^{\prime}\right)\right)$.

- If $T\left(s_{3}, q\right)=\left(s^{\prime}, N, q^{\prime}\right)$ :

$(\forall x \in A)(\forall y \in A)(\forall z \in A)\left(x \rightarrow y \rightarrow z \wedge \chi(x)=\diamond \wedge \chi(y)=\left(s_{2}, q\right) \wedge\right.$ $\left.\left(\left(\chi(z)=s_{3}\right) \vee(\chi(z)=\varnothing)\right) \Rightarrow y \rightarrow h(x, y, z) \wedge \chi(h(x, y, z))=\left(s^{\prime}, q^{\prime}\right)\right)$,

- If $T\left(s_{2}, q\right)=\left(s^{\prime}, L, q^{\prime}\right)$ or $\left(s^{\prime}, R, q^{\prime}\right)$ :

$(\forall x \in A)(\forall y \in A)(\forall z \in A)\left(x \rightarrow y \rightarrow z \wedge \chi(x)=\diamond \wedge \chi(y)=\left(s_{2}, q\right) \wedge\right.$ $\left.\left(\left(\chi(z)=s_{3}\right) \vee(\chi(z)=\varnothing)\right) \Rightarrow y \rightarrow h(x, y, z) \wedge \chi(h(x, y, z))=s^{\prime}\right)$.

- $(\forall x \in A)(\forall y \in A)(\forall z \in A)$ (all previous antecedents in this group are false $\Rightarrow h(x, y, z)=\infty)$.

\section{Acknowledgments}

The author is greatly indebted to Ross Willard for his invaluable comments and suggestions, and for many fruitful discussions, which lead to the final form of the paper.

Also, the author would like to express his gratitude to Anthony Bonato for a patient reading of the manuscript and comments which helped a better flow of the paper.

Finally, the author would like to thank the referee for many suggestions which contributed to a significant improvement of the manuscript from its original form.

\section{REFERENCES}

[1] E. Börger, Computability, Complexity, Logic, North-Holland, Amsterdam, 1989. MR 90h:68041

[2] S. Burris, Polynomial time uniform word problems, Math. Logic Quart., 41 (1995), 173-182. MR 96j:08004

[3] S. Burris and H. P. Sankappanavar, A Course in Universal Algebra, Graduate Texts in Mathematics, vol. 78, Springer-Verlag, New York, 1981. MR 83k:08001

[4] S. Crvenković and D. Delić, A variety with locally solvable but globally unsolvable word problem, Algebra Universalis, 35 (1996), 420-424. MR 97d:03058 
[5] O. Kharlampovich and M.V. Sapir, Algorithmic problems in varieties, Internat. J. Algebra Comput., 5 (1995), 379-602. MR 96m:20045

[6] D. Kozen, Complexity of finitely presented algebras, Proc. of the 9th Symposium, STOC, pages 164-177, 1977. MR 58:8487

[7] G. Kreisel and J.L. Krivine, Elements of Mathematical Logic, North-Holland, Amsterdam, 1967. MR 36:2463

[8] A.I. Mal'cev, On homomorphisms onto finite groups (in Russian), Učen. Zap. Ivan. Ped. Inst., 18 (1958), 49-60.

[9] E.W. Mayr and A.R. Meyer, The complexity of the word problem for commutative semigroups and polynomial ideals, Adv. in Math., 46 (1982), 305-329. MR 84g:20099

[10] R. McKenzie, On spectra, and the negative solution of the decision problem for identities having a nontrivial model, J. Symbolic Logic, 40 (1975), 186-196. MR 51:12499

[11] R. McKenzie, G. McNulty, and W. Taylor, Algebras, Lattices, Varieties, Vol.I, Wadsworth \& Brooks/Cole, Monterey, CA, 1987. MR 88e:08001

[12] R. McKenzie and M. Valeriote, The Structure of Locally Finite Decidable Varieties, Progress in Mathematics, vol. 79, Birkhäuser, Boston, 1989. MR 92j:08001

[13] G. McNulty, A field guide to equational logic, J. Symbolic Comp., 14 (1992), 371-397. MR 94g:03065

[14] A. Mekler, E. Nelson, and S. Shelah, A variety with solvable, but not uniformly solvable, word problem, Proc. London Math. Soc., 66 (1993), 225-256. MR 93m:03018

[15] A. Schmidt, Über deduktive Theorien mit mehreren Sorten von Grunddingen, Math. Ann., 115 (1938), 485-506.

[16] W. Taylor, Characterizing Mal'cev conditions, Algebra Universalis, 3 (1973), 351-397. MR 50:2030

[17] B. Wells, Pseudorecursive varieties of semigroups. I, Internat. J. Algebra Comput. MR 97k:20099

[18] B. Wells, Pseudorecursive Varieties and Their Implications for Word Problems. PhD thesis, University of California, Berkeley, 1982.

Department of Pure Mathematics, University of Waterloo, Waterloo, Ontario, N2L 3G1 CANADA

E-mail address: ddelic@math.uwaterloo.ca 